\title{
AFibNet: An Implementation of Atrial Fibrillation Detection With Convolutional Neural Network
}

\section{Bambang Tutuko}

Intelligent System Research Group, Faculty of Computer Science, Universitas Sriwijaya, Palembang, 30139, Indonesia

\section{Siti Nurmaini ( $\nabla$ sitinurmaini@gmail.com )}

Intelligent System Research Group, Faculty of Computer Science, Universitas Sriwijaya, Palembang, 30139, Indonesia

\section{Alexander Edo Tondas}

Department of Cardiology \& Vascular Medicine, Dr. Mohammad Hoesin Hospital, Palembang, Indonesia

Muhammad Naufal Rachmatullah

Intelligent System Research Group, Faculty of Computer Science, Universitas Sriwijaya, Palembang, 30139, Indonesia

\section{Annisa Darmawahyuni}

Intelligent System Research Group, Faculty of Computer Science, Universitas Sriwijaya, Palembang, 30139, Indonesia

\section{Ria Esafri}

Intelligent System Research Group, Faculty of Computer Science, Universitas Sriwijaya, Palembang, 30139, Indonesia

\section{Firdaus Firdaus}

Intelligent System Research Group, Faculty of Computer Science, Universitas Sriwijaya, Palembang, 30139, Indonesia

\section{Ade Iriani Sapitri}

Intelligent System Research Group, Faculty of Computer Science, Universitas Sriwijaya, Palembang, 30139, Indonesia

\section{Research Article}

Keywords: Cloud Deep Learning, 1D-Convolutional neural network, Atrial Fibrillation

Posted Date: February 15th, 2021

DOl: https://doi.org/10.21203/rs.3.rs-228165/v1

License: (c) (i) This work is licensed under a Creative Commons Attribution 4.0 International License. Read Full License 
Version of Record: A version of this preprint was published at BMC Medical Informatics and Decision Making on July 14th, 2021. See the published version at https://doi.org/10.1186/s12911-021-01571-1. 


\title{
AFibNet: An Implementation of Atrial Fibrillation Detection with Convolutional Neural Network
}

\author{
Bambang Tutuko ${ }^{1}$, Siti Nurmaini ${ }^{1 *}$, Alexander Edo Tondas ${ }^{2}$, Muhammad Naufal Rachmatullah ${ }^{1}$, Annisa \\ Darmawahyuni $^{1}$, Ria Esafri ${ }^{1}$, Firdaus Firdaus ${ }^{1}$ and Ade Iriani Sapitri ${ }^{1}$
}

\footnotetext{
${ }^{*}$ Correspondence:

sitinurmaini@gmail.com

${ }^{1}$ Intelligent System Research

Group, Faculty of Computer

Science, Universitas Sriwijaya,

Palembang, 30139, Indonesia

${ }^{2}$ Department of Cardiology

Vascular Medicine, Dr.

Mohammad Hoesin Hospital,

Palembang, Indonesia

Full list of author information is

available at the end of the article
}

\begin{abstract}
Background: Generalization model capacity of deep learning (DL) approach for atrial fibrillation (AF) detection remains lacking. It can be seen from previous researches, the DL model formation used only a single frequency sampling of the specific device. Besides, each electrocardiogram (ECG) acquisition dataset produces a different length and sampling frequency to ensure sufficient precision of the R-R intervals to determine the Heart Rate Variability (HRV). An accurate HRV is the gold standard for predicting the AF condition. Hence, we propose a DL approach to analyze massive amounts of ECG raw data in a broad range of devices to overcome a current challenge.

Results: This paper demonstrates powerful results for end-to-end implementation of AF detection based on a convolutional neural network (AFibNet). The method used a single learning system without considering the variety of signal lengths and frequency samplings. For implementation, the AFibNet is processed with a computational cloud-based DL approach. This study utilized a one-dimension convolutional neural networks (1D-CNNs) model for 11,842 subjects. It was trained and validated with 8,232 records based on three datasets and tested with 3,610 records based on eight datasets. The predicted results, when compared with the diagnosis results indicated by human practitioners, showed a $99.80 \%$ accuracy, sensitivity, and specificity. When tested with unseen data, the AF detection reaches $98.94 \%$ accuracy, $98.97 \%$ sensitivity, and $98.97 \%$ specificity in 0.02 seconds for one instance when processed in the DL-Cloud System.
\end{abstract}

Conclusions: These findings demonstrate that the proposed model approach can used in a broad range of devices and validated to unknown data to derive feature maps and reliably detect the AF periods. We have found that our cloud-DL system is suitable for practical deployment.

Keywords: Cloud Deep Learning; 1D-Convolutional neural network; Atrial Fibrillation

\section{Introduction}

A single pulse of an electrocardiogram (ECG) signal consists of the morphology, heart rate, regularity, wave segments, relative amplitudes, timing intervals, and normalized energy in a beat or a rhythm [1]. ECG is a popular non-invasive tool used to classify healthy and unhealthy cardiac activity based on a time series signal [2][3]. An estimated 300 million or more ECGs are recorded worldwide every year [2], representing a tremendous amount of data for cardiologists to analyze.

One electrophysiologic disturbance within the atria that can be observed by ECG is termed atrial fibrillation $(\mathrm{AF})[2]$. AF is the most prevalent severe abnormal heart 
rhythm associated with a fast heart rate. It refers to an abnormal, rapid, and nonsynchronized muscle fiber contraction with complex patophysiology [4][5][6][7], and is recognized as an independent risk factor for stroke, with important clinical and economic consequences. Diagnosing the symptoms is important before treatment of this severe disease; however, existing commercial ECG devices for AF detection methods still show actual misdiagnosis rates. This is largely due to the lack of generalizability caused by tuning only for specific medical devices [8].

Continuous or real-time monitoring of an ECG may help distinguish heart abnormalities. All ambulatory 12-lead ECG systems are designed to ensure reliable AF detection. In environments such as primary care centers and emergency units, where no experts are available to examine and interpret ECG tracings, these ECG devices are commonly used. Unfortunately, these devices are pricey, time-consuming, challenging to use, and require long-term exposure for AF measurements [9]. An automatic and accurate interpretation is critical in low and middle-income countries, and could potentially prevent $75 \%$ of cardiovascular disease deaths [9], as those populations often do not have access to cardiologists with full expertise in ECG diagnosis. In fact, medical professionals in those environments typically have limited diagnostic expertise in interpreting 12-lead ECGs [9][10]. Therefore, a simple AF detection that does not require hospital visits and is publicly accessible is required for better diagnosis.

The use of single-lead ECG with short-term detection is currently prevalent in daily applications because the device is simple, low cost, and easy to use [11]. Regardless, AF detection using short-term signal detection can be missed in many cases due to the lack of data standardization collection, the processing procedures used, and the inconsistent reporting of technological factors, such as frequency sampling [12][13]. Several short-term ECG instruments have variable signal quality, frequencies, and lengths for detecting AF episodes. Data may also need to be sampled at a different target frequency, when dealing with multiple instruments that are sampled at various frequencies. However, choosing data sampled at a certain frequency will influence the generalization potential and complexity of the model [13]. Therefore, the chosen approach must be robust without decreasing effectiveness of the device to detect AF [13]. Hence, a basic approach for enhancing short-term AF identification with acceptable results is desirable.

Many of the computer-aided ECG signals proposed for AF detection over the past 50 years are based on machine learning (ML) [14] and have been used in commercial ECG medical devices [15]. Two significant bottlenecks that still hinder early autodetection are the energy limitations of the continuous monitoring equipment and the lack of efficient ML-based models for AF prediction. In addition, conventional ML requires a separate technique of feature engineering that can be computationally expensive. The desired ML-based solution for automatic AF diagnosis therefore requires high accuracy but it also needs to be computationally efficient [16].

Recently, deep learning (DL) methods have shown great potential in the healthcare and medical areas [17][18]. Specifically, some pioneering work has shown success in using DL methods for AF detection [19][20][21]. DL models can be trained to perform beat and rhythm detection/classification using ECG data collections but, unfortunately, the use of DL for AF detection remains essentially unexplored [22]. 
One DL approach is to use convolutional neural networks (CNNs) architecture with feature engineering embedded into the learning structure. Actually, the CNNs is a type of DL that excels in processing 2D data, such as images. However, by considering signals as 1-dimensional (1D) data, studies have shown promising results using convolutions for signal processing [20][23][24][25]. 1D-CNNs show superiority in AF signal processing and have outperformed both recurrent neural networks (RNNs) and deep neural networks (DNNs) [13].

Previous studies have shown that 1D-CNNs can successfully provide fast and accurate classification of long-term ECG records. They can analyze the morphological characteristics and learn the slit variation of an input signal during a short-term ECG [13]. The 1D-CNNs model is developed for patient-specific ECG classification [26]. A nine-layer CNNs model for classifying five types of heartbeats from initial signals used an augmentation technique and had a precision of $94.03 \%$ [27]. A 1DCNNs model consisting of 33 convolutional layers based on a massive ECG dataset of 91,232 records from 53,549 patients was able to identify 12 rhythm categories [28]. A generic CNNs has been presented for patient-specific ECG classification [29]. The use of modified U-net architecture has been suggested to diagnose beatwise arrhythmia [30]. A 31-layer 1D residual CNNs model was developed to identify five different types of heartbeats [31]. A customized CNN model has been recommended to classify patient-specific heartbeats using 44 records [32]. A CNNs model has been applied for classification of 17 cardiac arrhythmias using long-duration ECG signals [33]. An end-to-end deep learning model has been proposed to classify 15 ECG classes [34]. However, despite this extensive study of the 1D-CNNs algorithm for classification/detection of ECG wave signals, the robustness of this algorithm remains an important issue and these methods are still far removed from practical applications [35].

Computerized ECG signal interpretation plays a critical role in the clinical workflow. Digital ECG data are readily accessible and the DL algorithmic model offers an opportunity to greatly increase the precision and scalability of automated ECG analysis [28]. A comprehensive evaluation of an end-to-end DL approach for AF ECG analysis across a wide variety of diagnostic devices has not been previously reported. None of the current models have been deployed to provide publicly available ECG AF detection services. Therefore, the aim of the present study was to propose a cloud-based 1D-CNNs approach that can be used to enhance AF detection based on CNNs by connecting it to the internet (AFibNet). This approach can provide easy and early detection of a potential AF anytime and anywhere. This study and the proposed approach make the following novel contributions:

- An end-to-end of AF detection with a broad range of distinct ECG devices

- Development of a generalization model for $1 \mathrm{D}-\mathrm{CNNs}$ into a single learning system named AFibNet

- Implementation of the proposed model in a cloud deep learning model with 11,842 subjects

- Validation of the robustness of $1 \mathrm{D}-\mathrm{CNNs}$ with a cloud system in preparation for publicly available ECG AF detection services.

The rest of this paper is organized as follows: Section 2 explains the method, and Section 3 presents the result and discussion. Finally, the conclusions are presented in Section 4. 


\section{Method}

Currently, health care information technology uses a cloud service to develop a system that combines medical devices and applications [24][36][37]. The use of these technologies connects patients to their physicians and facilitates the sharing of medical data over a safe network, thereby eliminating needless hospital visits and lessening the burden on the health care system [28]. The patients can measure their own heart conditions and the measurement results of ECG signal recording are delivered to a central storage location for centralized decision-making. These measurement data are usually physiological signals in the cardiac ECG signal domain, such as beat, rhythm, and HRV [38]. The patients use short-term ECG devices and transmit the HRV signals to a mobile device for relay to the cloud server. Figure 1 shows a framework for collecting and analyzing ECG device data from a cloud server. Once the ECG data are collected, they are transferred to the mobile terminal via Bluetooth and displayed in real time, then transmitted to the cloud through WIFI or 4G. The DL architecture then validates and analyzes the incoming HRV signals in real time. If the model detects $\mathrm{AF}$ in the signals, a cardiologist is informed. The cardiologist can then review the suspicious HRV trace as a beat or rhythm and reach a diagnosis. The diagnosis can later be communicated to the patient in a simple scheme.

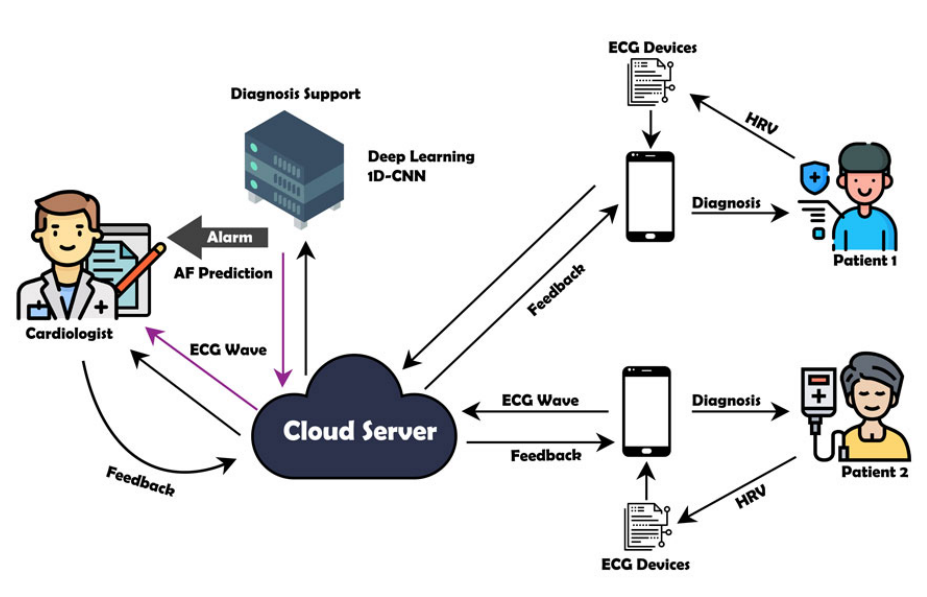

Figure 1 Flow diagram of deep learning model for AF diagnosis system

This work proposes only the DL-based cloud system with the 1D-CNNs model in the main processing system for AF diagnosis support. The cloud-based DL is important for the AF diagnosis as it provides an accurate medical interpretation system. To ensure that the implementation works properly in all stages, all parts of the DL-based cloud system design are presented as follows.

\section{Dataset}

In this study, nine public ECG databases are utilized: the MIT-BIH Atrial Fibrillation [39], the 2017 PhysioNet/CinC Challenge [40], the China Physiological Signal Challenge 2018 [41], ECG Long Term AF [42], Paroxysmal AF [40], AF Termination Challenge [43], Fantasia [44], MIT-BIH Arrhythmia [45], and ECG recording from 
Chapman University and Shaoxing People's Hospital [46], as well as two ECG signal recording databases collected from an Indonesian Hospital. Based on all these datasets, we separated three main processes: training, validating, and testing. In this process, all data sets differed in the lengths of signal recording and frequency sampling.

Three datasets (MIT-BIH Atrial Fibrillation, the 2017 PhysioNet/CinC Challenge, and the China Physiological Signal Challenge 2018 databases) were used for training and validating. The model then was tested using the other eight datasets. The summary and samples of the ECG dataset used in this study are shown in Table 1 and Figure 2. All data were collected from a short single-lead recording with different ECG devices, with a length of signal recording varying from 10 seconds to 25 hours and frequency varying from 100 to $500 \mathrm{~Hz}$. The ECG data from 11,842 subjects can be described as follows:

\section{- MIT-BIH Atrial Fibrillation}

This database has 23 public ECG recordings taken from AF patients every 10 hours. The ECG signals were sampled at $250 \mathrm{~Hz}$, with four types of rhythm annotations, (AF, atrial flutter $[\mathrm{AFL}], \mathrm{AV}$ junctional rhythm $[\mathrm{J}]$, and all other rhythms $[\mathrm{N}])$. The analog ECG recordings were made at the Beth Israel Deaconess Medical Center using ambulatory ECG recorders with a typical recording bandwidth of approximately $0.1 \mathrm{~Hz}$ to $40 \mathrm{~Hz}$.

- The 2017 PhysioNet/CinC Challenge

All ECG records were sampled at $250 \mathrm{~Hz}$ by a single lead with four types of rhythms (N, AF, non-AF [NAF; other arrhythmias], and Noisy). We selected the recordings only from $\mathrm{N}$ and AF. All ECG recordings were collected using the AliveCor device for 9 to 60 seconds.

- The China Physiological Signal Challenge 2018

This database was collected from 11 hospitals sampled at $500 \mathrm{~Hz}$, with normal and abnormal types. All 12-lead ECG recordings lasted from 6 to 60 seconds and were taken from 3178 female and 3699 male patients. The present study used only single lead (Lead II) data with two rhythms (N and AF), with 981 and 1,098 total records, respectively.

- ECG Long Term AF

This database has 84 long-term ECG recordings of subjects with paroxysmal or sustained AF. Each record was digitized at $128 \mathrm{~Hz}$, and the durations vary but are typically 24 to 25 hours. The 38 records indicated as AF termination were utilized in the present study. The original recordings were digitized and automatically annotated at Boston's Beth Israel Deaconess Medical Center. Steven Swiryn and George Moody annotated the AF terminations.

- Paroxysmal AF

This challenge database consists of 50 pairs of half-hour ECG recordings sampled at $128 \mathrm{~Hz}$. The database consists of group A, who experienced PAF and group $\mathrm{N}$ who did not have PAF. We have tested group A only, with a total of 48 records.

- MIT-BIH Arrhythmia

The database was digitized at 360 samples per second and contains 48 halfhour excerpts of two-channel ambulatory ECG recordings. The database was 
obtained from 47 subjects studied by the BIH Arrhythmia Laboratory between 1975 and 1979. The database has two types: beats and rhythms. This study tested the AF rhythm type of the ECG recordings (records 201, 203, 210, 217, 219, and 221).

- AF Termination Challenge

This database is divided into a learning set and two test sets. The learning set contains 30 records in total, with 10 records in each of three groups $(\mathrm{N}, \mathrm{S}$, and T). Each record was sampled at $128 \mathrm{~Hz}$, and the segments were extracted from 20-24 hour ECG recordings. The ECG recordings were created for use in the Computers in Cardiology Challenge 2004. Among the three groups, the present study used group $\mathrm{T}$, in which the AF terminates immediately.

\section{- Fantasia}

All ECG recordings of sinus rhythm subjects were digitized at $250 \mathrm{~Hz}$. Each set includes the respiration belt data from 20 young (21-34 years old) and 20 elderly (68-86 years old) subjects. For unseen data testing, the present study used only 24 records randomly chosen from the young and elderly cohorts. The respiration signals were collected by 120 minutes of continuous supine resting while under continuous ECG.

- ECG recording from Chapman University and Shaoxing People's Hospital This database includes a large number of individual subjects (more than 10,000) with 12-lead ECG signals sampled at a higher than usual sampling rate of $500 \mathrm{~Hz}$. The database includes 11 heart rhythms and 56 types of cardiovascular conditions labelled by professional physicians. The ECG records were acquired over 10 seconds. The ECG recordings were collected from 10,646 patients, including 5,956 males and 4,690 females. In the present study, we utilized only 1,780 AF data and 1,826 Normal data selected from Lead II for unseen data.

- ECG recording from an Indonesian Hospital

The Indonesian Hospital dataset contained 42 and 13 records sampled at 500 $\mathrm{Hz}(\mathrm{N}$ and $\mathrm{AF})$ and $400 \mathrm{~Hz}(\mathrm{AF})$, respectively. All ECG records were collected for 10 seconds. The ECG database was collected by clinicians from patients who use ambulatory ECG devices (February to June 2020). 
Table 1 ECG raw data description

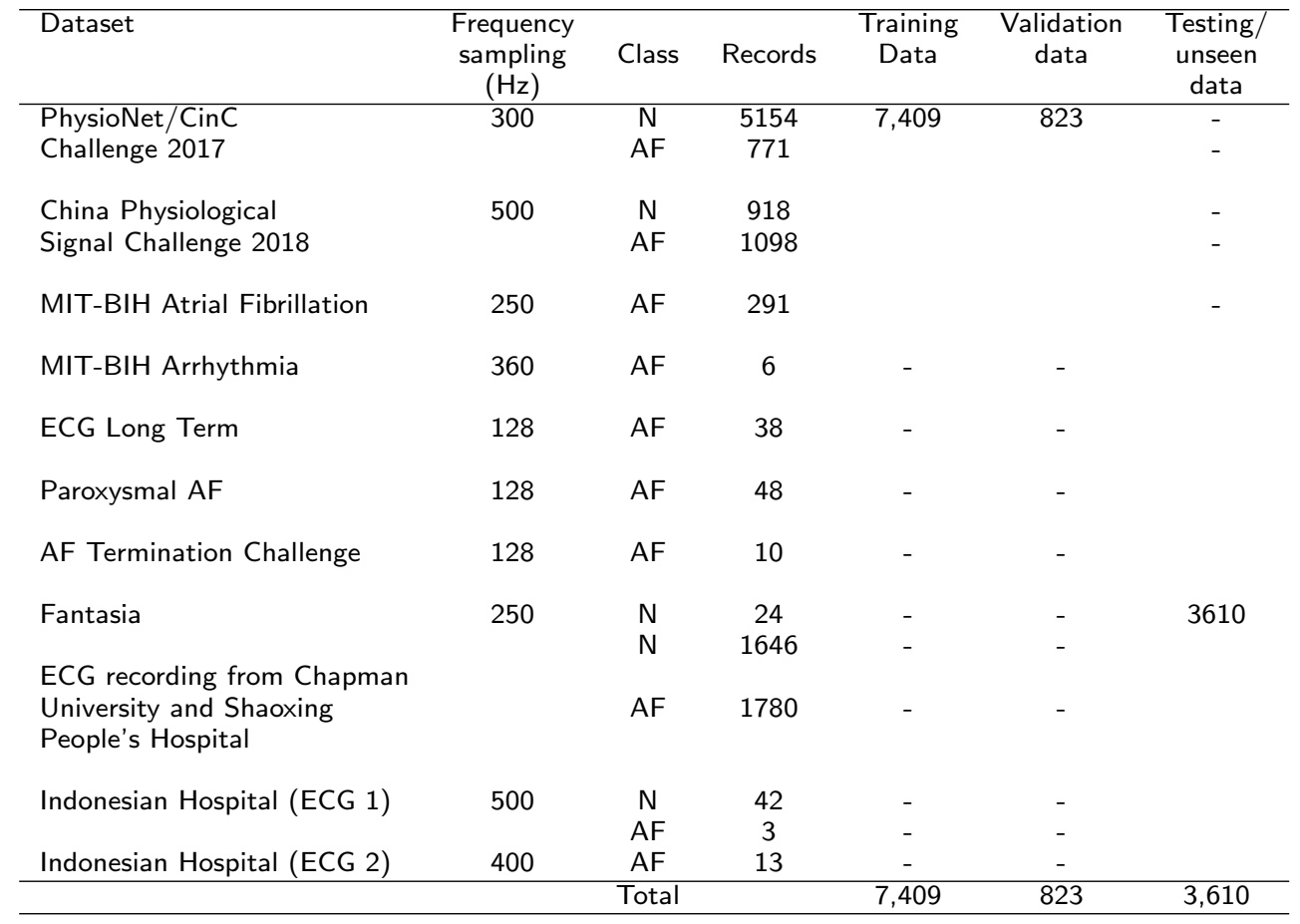
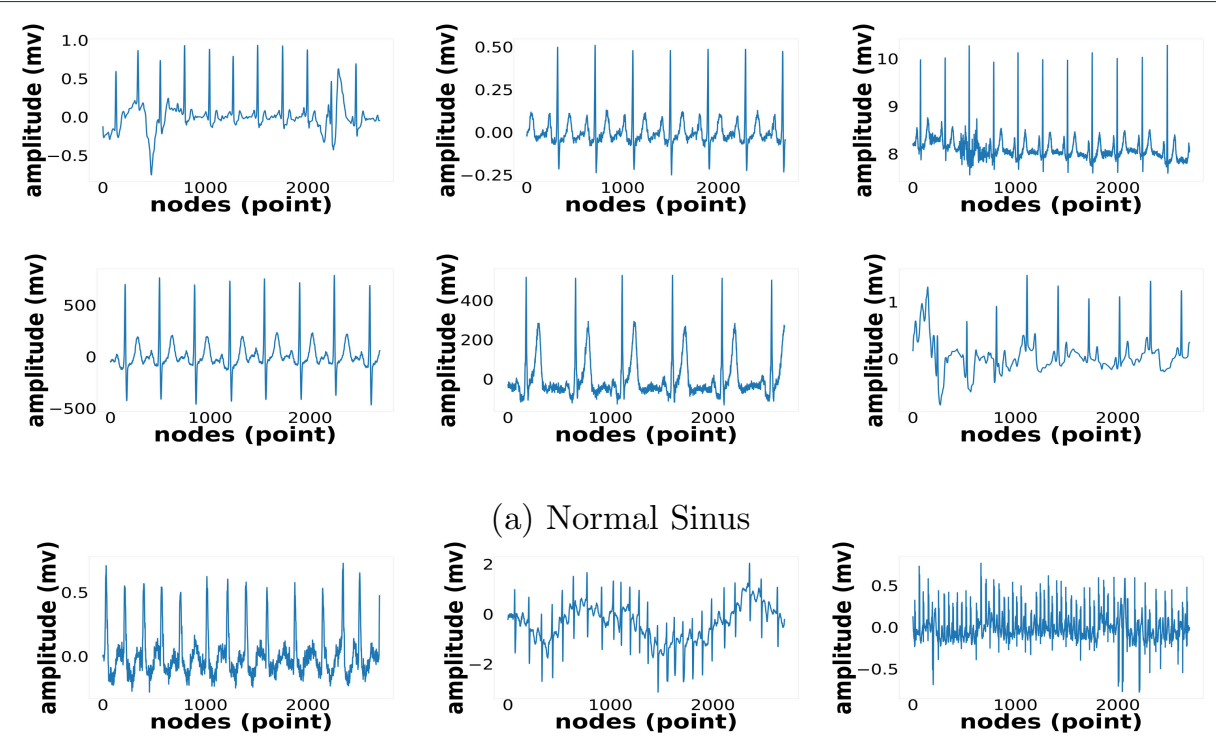

(a) Normal Sinus
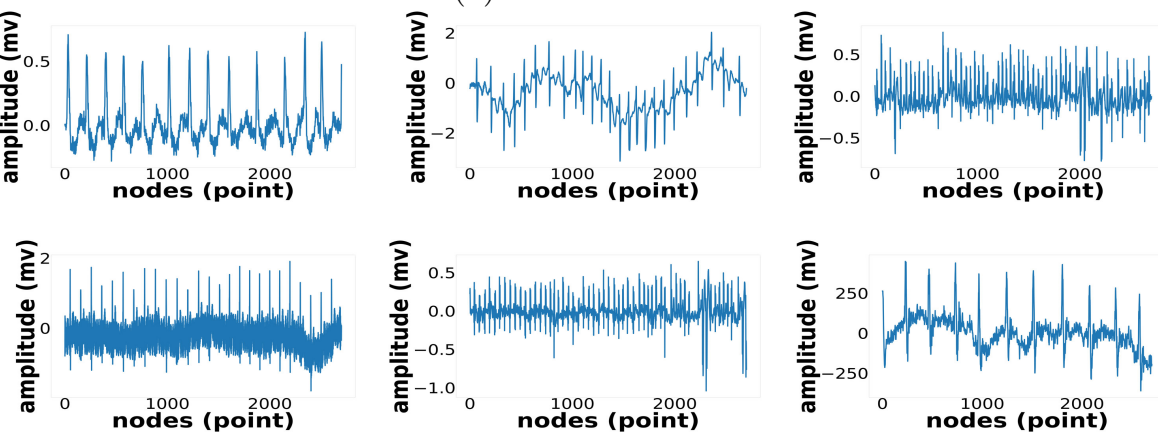

(b) Atrial Fibrillation

Figure 2 Flow diagram of deep learning model for AF diagnosis system 


\section{Implementation of Atrial Fibrillation Detection}

In this study, our classifier is based on 13 convolution and 5 max-pooling layers of ID-CNNs architecture, which we have published in detail in a previous work [13]. To assure the classifier performance, in the present work, the numbers of data trainings and validations are increased to 8,232 records. The proposed model takes an input from several devices with different frequency samplings and lengths of the ECG signal waveform. The training process uses 3 datasets: PhysioNet/CinC sampling at $300 \mathrm{~Hz}$, China Physiological at $500 \mathrm{~Hz}$, and MIT-BIH Atrial Fibrillation at 250 Hz. Figure 3 shows the explainable of AFibNet methodology, which consisted of the following six main steps as follow;

- In this work, we use large ECG data of the $\mathrm{N}$ and AF condition from 11,842 subjects collected from eleven datasets for training, validating, and testing. We use inter-patiens mechanishm, where the training/validating dataset is separate with testing dataset. All data are taken from single-lead ECG standard recordings with varying lengths of signal and frequency sampling (100-500 $\mathrm{Hz})$.

- ECG noise removal using discrete wavelet transform (DWT). This step decompose the ECG signals into the specific wavelet levels (8 levels) with Sym5 [13]. The signal frequency is divided by two in DWT because it passes through the high pass and low pass filters. Frequencies that pass through the high pass filter will enter the detail coefficient, while the low pass filter will enter the approximation coefficient.

- All ECG signals are segmented into 2700 samples for one episode. An AF condition may contract at up to 600 beats per minute (bpm), thereby creating a high irregularity of R-R intervals and a sudden disappearance of regularly occurring P-waves [47][39]. Therefore, at least three to four heartbeats are needed to represent the AF episodes [39]. To assess the R-R interval in all ECG records, we have considered the minimum and maximum lengths for ECG signal segmentation based on the training datasets mentioned earlier. The ECG segmentation of 2700 nodes contained at least two R-R intervals or three beats with different frequency samplings $(250 \mathrm{~Hz}, 300 \mathrm{~Hz}$, and 500 $\mathrm{Hz}$ ) in all records. In addition, with a minimum frequency sampling of $128 \mathrm{~Hz}$ for the testing set, the 2700 nodes segmentation could present more than two R-R intervals. Hence, 2700 nodes for ECG segmentation were selected as the best ECG episodes.

- Two features are generated from ECG signal irregularly irregular of heart rhythm and the maksimum of amplitude as R-peak in one episodes of ECG signal, and it learn episode by episode. The feature is represented by ECG signal amplitude along 2700 nodes. The most important process of the 1DCNNs method is that the common cause of AF is modeled by a series of filters in the convolution layer and sub sampling in the maxpooling layer. The feature output is used to synthesize the corresponding potential abnormal and normal rhythms. The feature reduce from 2700 nodes in layer-1 becomes 78 nodes in layer-13 with maxpooling-5, and the selected feature that use as input in fully connected layer to classify the normal and AF feature.

- Each ECG signal episodes of 2700 nodes was trained using the 1D-CNNs classifier model was proposed by Nurmaini et al. [13]. The structure model has 13 
hidden layers with an activation function rectified linear unit (ReLU) in the hidden layers and tanh-sigmoid in the output layers [13]. The hyperparameters utilize a 0.0001 learning rate, 16 batch size, and 100 epochs. The training process for AF detection was fully supervised. It back-propagated the gradients from the fully connected layer through to the convolutional layers. As a loss function, we minimized the binary cross-entropy to optimize the model parameters, and we utilized the gradient descent with the Adam update rule.

- The 1D-CNNs model was proposed with several hardware platforms and software frameworks using both local (on-device) and remote (network-side server) computation (refer to Figure 1). The DL-based cloud system is designed to process the AF detection and to ensure that the proposed model works properly in real applications. Therefore, the computational complexity is deeply analyzed. Three parameters of the computational complexity as a cloud performances, namely processing time, throughput, and testing time, are validated using a computer with and without GPU. The memory consumption is one of the parameters to be considered, based on the selected classifier model. Each process in the convolution layer that is fully connected can be counted as memory consumption in our model and can be calculated from output shape from each layer parameter in the CNNs architecture.

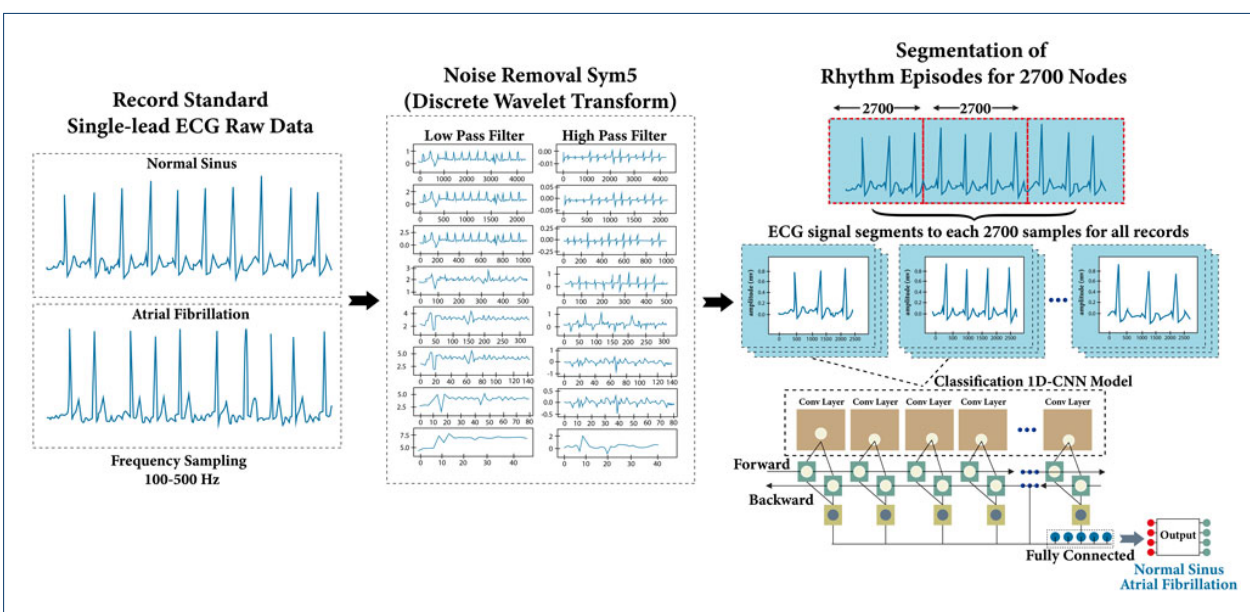

Figure 3 Proposed the AFibNet for explainable Al methodology

\section{Explainable Feature Learning in 1D-CNNs}

1D-CNNs architecture has two distinct layer types, followed by CNNs-layer and then fully-connected (FC) layer. The feature learning was processed in CNNs-layer by using convolution and sub-sampling (pooling) process. The specific function of the two layers is for reducing the complexity and dimension of the ECG feature. In this study, we generate one by one episode (2700 nodes) as a feature along with ECG signal recording. The amplitude is represented by each node from node 1 to 2700 . The CNN-layers process and learn to extract features (feature learning) of the raw 1D data, which are used in the classification task performed by the FC-layersAs a result, both feature extraction and classification operations are integrated into one 
process that can be streamlined to improve the performance of the classification. All feature learning process is explained detail in Table 2 .

In the convolution process, several unique features are generating. For example, in convolution layer 1, was feed with 2700 nodes of the ECG signal. This layer has 64 kernels with a size of $3 \times 1$ and a stride of 1 . This striding process is carried out along the ECG signal so that it can produce 3 features at the beginning, middle, and end of the signal episode. Then, the result of the convolution process is continued to the max-pooling layer. The pooling layer aims to summarize the features resulting from the convolution process so that it not only reduces the computation load but also can strengthen the model against variations in the input signal.

Table 2 Feature learning interpretation

\begin{tabular}{|c|c|c|c|c|c|}
\hline Layer & $\begin{array}{l}\text { Input } \\
\text { Nodes }\end{array}$ & $\begin{array}{l}\text { Filter } \\
\text { Number }\end{array}$ & $\begin{array}{l}\text { Kernel } \\
\text { Size/Pool } \\
\text { Size }\end{array}$ & $\begin{array}{l}\text { Output } \\
\text { Nodes }\end{array}$ & $\begin{array}{l}\text { Feature } \\
\text { Interpretation }\end{array}$ \\
\hline Input & 2700,1 & - & & - & $\begin{array}{l}\text { ECG amplitude for } \\
\text { one episode }\end{array}$ \\
\hline Convolution 1 & 2700,1 & 64 & $3 \times 1$, stride 1 & $2698 \times 64$ & 64 feature map \\
\hline Convolution 2 & $2698 \times 64$ & 64 & $3 \times 1$, stride 1 & $2696 \times 64$ & 64 feature map \\
\hline Max-pooling 1 & $2696 \times 64$ & - & $2 \times 1$, stride 2 & $1348 \times 64$ & $\begin{array}{l}\text { Feature reduction } \\
\text { ( } 1348 \text { nodes for } \\
\text { one episode) }\end{array}$ \\
\hline Convolution 3 & $1348 \times 64$ & 128 & $3 \times 1$, stride 1 & $1346 \times 128$ & 128 feature map \\
\hline Convolution 4 & $1346 \times 128$ & 128 & $3 \times 1$, stride 1 & $1344 \times 128$ & 128 feature map \\
\hline Max-pooling 2 & $1344 \times 128$ & - & $2 \times 1$, stride 2 & $672 \times 128$ & $\begin{array}{l}\text { Feature reduction } \\
\text { ( } 672 \text { nodes for } \\
\text { one episode) }\end{array}$ \\
\hline Convolution 5 & $672 \times 128$ & 256 & $3 \times 1$, stride 1 & $670 \times 256$ & 256 feature map \\
\hline Convolution 6 & $670 \times 256$ & 256 & $3 \times 1$, stride 1 & $668 \times 256$ & 256 feature map \\
\hline Convolution 7 & $668 \times 256$ & 256 & $3 \times 1$, stride 1 & $666 \times 256$ & 256 feature map \\
\hline Max-pooling 3 & $666 \times 256$ & - & $2 \times 1$, stride 2 & $333 \times 256$ & $\begin{array}{l}\text { Feature reduction } \\
\text { ( } 672 \text { nodes for } \\
\text { one episode) }\end{array}$ \\
\hline Convolution 8 & $333 \times 256$ & 512 & $3 \times 1$, stride 1 & $331 \times 512$ & 512 feature map \\
\hline Convolution 9 & $331 \times 512$ & 512 & $3 \times 1$, stride 1 & $329 \times 512$ & 512 feature map \\
\hline Convolution 10 & $329 \times 512$ & 512 & $3 \times 1$, stride 1 & $327 \times 512$ & 512 feature map \\
\hline Max-pooling 4 & $327 \times 512$ & - & $2 \times 1$, stride 2 & $163 \times 512$ & $\begin{array}{l}\text { Feature reduction } \\
\text { (163 nodes for } \\
\text { one episode) }\end{array}$ \\
\hline Convolution 11 & $163 \times 512$ & 512 & $3 \times 1$, stride 1 & $161 \times 512$ & 512 feature map \\
\hline Convolution 12 & $161 \times 512$ & 512 & $3 \times 1$, stride 1 & $159 \times 512$ & 512 feature map \\
\hline Convolution 13 & $159 \times 512$ & 512 & $3 \times 1$, stride 1 & $157 \times 512$ & 512 feature map \\
\hline Max-pooling 5 & $157 \times 512$ & - & $2 \times 1$, stride 2 & $78 \times 512$ & $\begin{array}{l}\text { Feature reduction } \\
\text { ( } 78 \text { nodes for } \\
\text { one episode) }\end{array}$ \\
\hline Flatten & 39936 & - & - & - & $\begin{array}{l}\text { Dot product } \\
\text { between } 78 \text { nodes } \\
\text { and } 512 \text { feature map }\end{array}$ \\
\hline Dense & - & - & - & 1000 & Weight params \\
\hline Dense & - & - & - & 1000 & Weight params \\
\hline Output & - & - & - & 1 & Class \\
\hline
\end{tabular}

\section{Result and Discussion}

\section{D-CNNs Classifier Performances}

Based on the data distribution, 6072 and 2160 records for $\mathrm{N}$ and AF signals were used in the training and validation process. All ECG data (about 8,232 records), after segmentation at 2700 for each record, produced 54,038 rhythm episodes. In order to avoid overfitting before the 1D-CNNs model was generated, a k-fold $(\mathrm{k}=10)$ validation technique was used to tune the class weight with the resampling procedure for the total data bias [13]; the performances reported in the results are the 
average scores. All data are split as $90 \%$ for the training process and the remainder for the validation process and are resampled tenfold again. Table 3 shows the data distribution of the $\mathrm{N}$ and $\mathrm{AF}$ condition data in each fold's training and validation set.

Table 3 Data segementation with a 10-fold scheme for a combination of three datasets (MIT-BIH Atrial Fibrillation, the 2017 PhysioNet/CinC Challenge, the China Physiological Signal Challenge 2018 databases)

\begin{tabular}{cccccc}
\hline \multirow{2}{*}{ Fold } & \multicolumn{2}{c}{ Training data } & \multicolumn{2}{c}{ Validation data } & Total \\
\cline { 2 - 5 } & $\mathrm{N}$ & $\mathrm{AF}$ & $\mathrm{N}$ & $\mathrm{AF}$ & \\
\hline 1 & 16485 & 32149 & 1790 & 3614 & 54038 \\
2 & 16391 & 32243 & 1884 & 3520 & 54038 \\
3 & 16424 & 32210 & 1851 & 3553 & 54038 \\
4 & 16402 & 32232 & 1873 & 3531 & 54038 \\
5 & 16469 & 32165 & 1806 & 3598 & 54038 \\
6 & 16520 & 32114 & 1755 & 3649 & 54038 \\
7 & 16476 & 32158 & 1799 & 3605 & 54038 \\
8 & 16453 & 32181 & 1822 & 3582 & 54038 \\
9 & 16416 & 32219 & 1859 & 3544 & 54038 \\
10 & 16439 & 32196 & 1836 & 3567 & 54038 \\
\hline
\end{tabular}

Each fold has obtained the 1D-CNNs model performance based on five metrics: accuracy, sensitivity, specificity, F-score, and precision (refer to Table 4). Overall, the model has obtained good performance with up to $99.80 \%$ accuracy. However, among the 10-fold cross validations, the 4th, 8th, and 9th fold achieved $100 \%$ in all performance metrics. This means the best folds of 4, 8, and 9 were entirely successful in classifying $\mathrm{N}$ and AF. The average accuracy, sensitivity, specificity, F-score, and precision for the two classes (N and AF) in tenfold were 99.8\%, 99.8\%, $99.8 \%, 99.77 \%$, and $99.74 \%$, respectively.

Table 4 Proposed 1D-CNNs model performance with 10-fold cross validation

\begin{tabular}{cccccc}
\hline \multirow{2}{*}{ Fold } & \multicolumn{5}{c}{ Classifier performances (\%) } \\
\cline { 2 - 6 } & Accuracy & Sensitivity & Specificity & F1-score & Precision \\
\hline 1 & 98.22 & 98.24 & 98.24 & 97.98 & 97.74 \\
2 & 99.94 & 99.94 & 99.94 & 99.93 & 99.93 \\
3 & 99.98 & 99.98 & 99.98 & 99.97 & 99.97 \\
4 & 100 & 100 & 100 & 100 & 100 \\
5 & 99.96 & 99.97 & 99.97 & 99.95 & 99.94 \\
6 & 99.98 & 99.98 & 99.98 & 99.97 & 99.97 \\
7 & 99.94 & 99.94 & 99.94 & 99.93 & 99.93 \\
8 & 100 & 100 & 100 & 100 & 100 \\
9 & 100 & 100 & 100 & 100 & 100 \\
10 & 99.98 & 99.98 & 99.98 & 99.97 & 99.97 \\
Average & 99.8 & 99.8 & 99.8 & 99.77 & 99.74 \\
\hline
\end{tabular}

The optimum ECG sampling rate required for HR analysis to ensure acceptable accuracy of R-R intervals has not yet been determined [48][49]. Previous studies indicate that a low sampling rate may decrease the accuracy in detection of R-R points, thereby changing the HR parameters [48][49]. In the present study, the proposed 1-D CNNs model has been tested with various datasets that vary in frequency sampling and lengths of the ECG signal recordings. No duplication exists between the training and testing sets, because they were separated at the beginning of process. Our proposed model therefore overcomes this problem and the ECG signal is still recognized as the $\mathrm{N}$ or $\mathrm{AF}$ condition.

The performance of the cloud DL approach is listed in Table 5 with the interval of frequency sampling of the ECG devices from $100 \mathrm{~Hz}$ to $500 \mathrm{~Hz}$. The proposed 1D-CNNs model has obtained perfect results for N and AF detection with $100 \%$ for 
accuracy, sensitivity, and specificity. The results show good performance without considering the frequency sampling.

Table 5 All performance of the AFibNet with several datasets

\begin{tabular}{|c|c|c|c|c|c|}
\hline \multirow[t]{2}{*}{ Data set } & \multirow[t]{2}{*}{ Class } & \multirow{2}{*}{$\begin{array}{l}\text { Number of } \\
\text { subjects }\end{array}$} & \multicolumn{3}{|c|}{ Performance (\%) } \\
\hline & & & Accuracy & Sensitivity & Specificity \\
\hline \multicolumn{6}{|c|}{ Training and Validation Data } \\
\hline $\begin{array}{l}\text { - The } 2017 \text { PhysioNet/ } \\
\text { CinC Challenge } \\
\text { - China Physiological }\end{array}$ & $\mathrm{N}$ & & & & \\
\hline $\begin{array}{l}\text { Signal Challenge } 2018 \\
\text { - MIT-BIH Atrial Fibrillation }\end{array}$ & AF & 8,232 & 99.8 & 99.8 & 99.8 \\
\hline \multicolumn{6}{|c|}{ Unseen Data Testing } \\
\hline ECG Long Term AF & $\mathrm{AF}$ & 38 & 100 & 100 & - \\
\hline Paroxysmal AF & $\mathrm{AF}$ & 48 & 100 & 100 & - \\
\hline MIT-BIH Arrhythmia & $\mathrm{AF}$ & 6 & 100 & 100 & - \\
\hline AF Termination Challenge & $\mathrm{AF}$ & 10 & 100 & 100 & - \\
\hline Fantasia & $\mathrm{N}$ & 24 & 100 & 100 & - \\
\hline \multirow{2}{*}{ Indonesian Hospital (ECG 1) } & $\mathrm{N}$ & 42 & 100 & 100 & 100 \\
\hline & $\mathrm{AF}$ & 3 & & & \\
\hline $\begin{array}{l}\text { Indonesian Hospital (ECG 2) } \\
\text { ECG recording from Chapman }\end{array}$ & $\mathrm{AF}$ & 13 & 100 & 100 & - \\
\hline \multirow{2}{*}{$\begin{array}{l}\text { University and Shaoxing } \\
\text { People's Hospital }\end{array}$} & $\mathrm{N}$ & 1,646 & 98.86 & 98.88 & 98.88 \\
\hline & $\mathrm{F}$ & 1,780 & & & \\
\hline \multirow[t]{2}{*}{ All unseen data testing } & N & 1,712 & 98.94 & 98.97 & 98.97 \\
\hline & AF & 1,898 & & & \\
\hline
\end{tabular}

The methods of AF detection are mainly based on R-R intervals, short-term heart rate variability analysis, and sequential review to verify the presence of $\mathrm{P}$-waves. In this work, the procedure has been tested on healthy subjects with $\mathrm{N}$ in order to detect any false positive (FP) and false negative (FN) predictions as a way to clarify the robustness of the technique. As shown in Table 5, when the data are increased, the proposed model of misclassified data shows that the classifiers produce $10 \mathrm{FN}$ and $29 \mathrm{FP}$ that impact the approach output. The potential solution to this problem is to remove the noise level in the ECG signal with other filters to maximize the method's efficiency. In the future, the preprocessing step will be improved in terms of a filter fusion mechanism for noise removal from the ECG recordings.

\section{Validating Robustness in a Cloud Server}

A feature map used as new input data for the next step was generated by the convolution process. In the $1 \mathrm{D}-\mathrm{CNNs}$ model with only simple array operations, the network learns $1 \mathrm{D}$ signals with a few hidden layers and neurons. After the nonlinearity process, all characteristics are produced and only during each convolution process; this stage never occurs in the pooling process. This allowed the model to create 64 unique features on the network's first layer. Due to its ability to avoid the vanishing gradient in the training process, we use ReLU as a nonlinearity function. The product of the layer of convolution is called the map of the function. We added the pooling layer after the second convolution layer. The purpose of this layer is to reduce the size of the feature map to lower the complexity. The max pooling layer is used since it can extract the essential features from the feature map. Two hidden layers in the fully connected part are created, each with 1000 nodes, while the output layer defines a sigmoid function to classify the ECG data. 
At present, the computing scene has become very diverse regarding computing platforms. A number of unique accelerators have been created, in addition to the rich variety of GPUs available for CNNs computations. The size ranges from small low-power systems to computing on the warehouse scale [50]. Meanwhile, the CPU development [13] has continued and many CPUs offer acceleration for CNNs computations. The same diversity applies to runtime systems [42]. The computational specification and performance of CNNs for AF detection are not yet well understood. In order to calculate the computational complexity of a CNN, the original implementation of the CNNs algorithm is needed. In the present study, the Keras library is utilized to implement the CNNs algorithm based on parallel processing for the training process so that it is unable to present the exact complexity of the CNNs algorithm. However, the weight parameters for every layer are calculated to predict the computational consumption. Our proposed 1D-CNNs model has 13 convolution layers with 5 polling layers, and the consecutive layers produce about 45 , 846, 329 weight parameters. All parameters are depicted in Table 6 . However, the only operation with a significant cost is a sequence of $1 \mathrm{D}$ convolutions which are simply linear weighted sums of two 1D arrays. Such a linear operation during the forward and backward operations can effectively be executed in parallel. It means although the parameters are a lot, it does not increase the computation time and resources.

The 1D-CNNs model is analyzed to determine the speed of the processing time needed to predict the ECG signal from the raw data and arrive at a decision in the cloud system. The whole process is divided into four stages: read data, denoising, load model, and inference. The computational consumption mostly involves the load model and inference stages. While the number of weight parameters is high, the execution time is quite negligible. For each relation, only scalar weight multiplication and addition are performed.

Table 6 The number of parameters produce based on 1D-CNNs architecture to show the computational complexity.

\begin{tabular}{lll}
\hline Layer Name & Output shape & Parameters \\
\hline Convolution 1 & (None, 2698, 64) & 256 \\
Convolution 2 & (None, 2696, 64) & 12352 \\
Maxpooling 1 & (None, 1348, 64) & 0 \\
Convolution 3 & (None, 1346, 128) & 24704 \\
Convolution 4 & (None, 1344, 128) & 49280 \\
Maxpooling 2 & (None, 672, 128) & 0 \\
Convolution 5 & (None, 670, 256) & 98560 \\
Convolution 6 & (None, 668, 256) & 196864 \\
Convolution 7 & (None, 666, 256) & 196864 \\
Maxpooling 3 & (None, 333, 256) & 0 \\
Convolution 8 & (None, 331, 512) & 393728 \\
Convolution 9 & (None, 329, 512) & 786944 \\
Convolution 10 & (None, 327, 512) & 786944 \\
Maxpooling 4 & (None, 163, 512) & 0 \\
Convolution 11 & (None, 161, 512) & 786944 \\
Convolution 12 & (None, 159, 512) & 786944 \\
Convolution 13 & (None, 157, 512) & 786944 \\
Maxpooling 5 & (None, 78, 512) & 0 \\
Flatten & (None, 39936) & 0 \\
Dense & (None, 1000) & 39936000 \\
Dense & (None, 1000) & 1001000 \\
Class & (None, 1) & 1001 \\
\hline Total of parameters: & & $45,846,329$ \\
\hline
\end{tabular}


This paper investigates the computational behavior and performance of AF detection from short-term ECG signals using 1D-CNNs. Table 7 lists the four computer specifications in the cloud in this work, and the testing is conducted for predicting the AF condition in unknown data from short-term ECG signals using several datasets. Using the 4th computer specification (refer to Table 7) and utilizing the GPU memory, a prediction of an AF condition takes 0.02 seconds. This means that high specifications for the CPU and GPU result in faster processing in the cloud system.

The throughput time, inference time, and memory consumption are also calculated in this work. The throughput is the number of instances that can be transmitted in one second on the network. We would like to process a single instance in as many instances as possible in parallel to reach the optimum throughput. A good rule of thumb is to hit the memory limit of the GPU for the specified data form to find the best network. This size depends on the type of hardware and the network size. As shown in the results, the entire AF classification phase can still be processed with good performance, and low computational complexities are provided by the 1D-CNNs at acceptably low cost with low power hardware.

Table 7 The sample of CPU and GPU process as a cloud server

\begin{tabular}{cllc}
\hline Specification & CPU & GPU & Testing (second) \\
\hline 1 & CPU1: 4 Core, 8 thread, Q2.8 GHz & - & 0.30 \\
& Memory: 16 Gb, Disk: 1000 Gb. & & 0.18 \\
2 & $\begin{array}{l}\text { CPU1: 4 Core, 8 thread, Q2.8 GHz } \\
\text { Memory: 16 Gb, Disk: 1000 Gb }\end{array}$ & GPU: GTX & 0.14 \\
3 & $\begin{array}{l}\text { CPU2: 8 Core, 16 thread, Q3.6 GHz } \\
\text { Memory: 32 Gb, Disk: 1000 Gb }\end{array}$ & \\
4 & $\begin{array}{l}\text { CPU2: 8 Core, 16 thread, Q3.6 GHz } \\
\text { Memory: 32 Gb, Disk: } 1000 \mathrm{~Gb}\end{array}$ & GPU2: RTX & 0.02 \\
& & & \\
\hline
\end{tabular}

Figure 4 illustrates the total time for the model to inference the input data. This process consists of three main processes: data reading, denoising, and inferencing. The processes of reading the data and denoising show no significant time differences among the four servers. However, when entering the inference step, servers equipped with GPUs have faster processing times compared to servers without GPUs. The time difference is quite striking due to the ability of the GPU to parallelize the process during the inference step. Overall, servers with CPU $2+$ GPU 2 specifications have the fastest processing times compared to the others. 


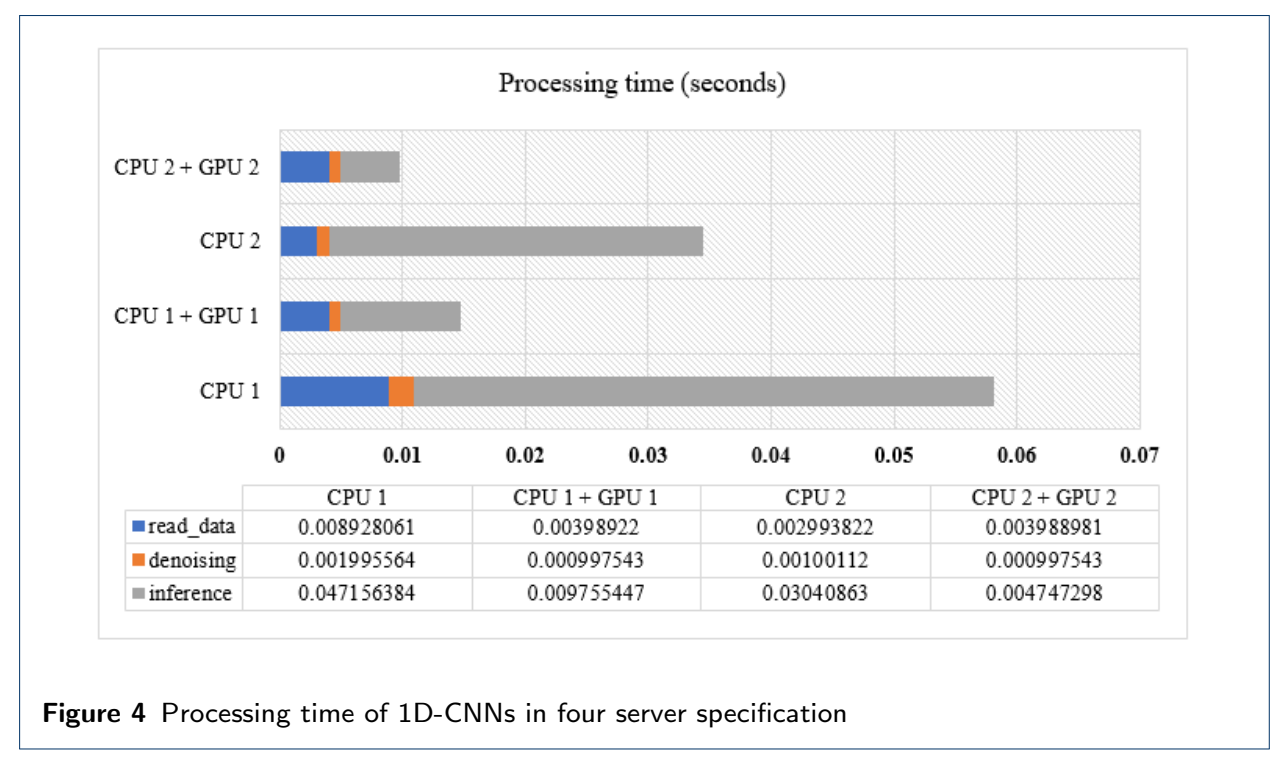

Another aspect that is also quite important in analyzing the robustness of a cloud server is the processing time for loading the model. Although this process is only done once (when the model is deployed to the server), this step is also quite important because the size of the DL model is relatively large and the processing time also takes time. In this paper, the size of DL model is more than $500 \mathrm{Mb}$. As shown in Figure 5, the server with CPU $2+$ GPU 2 has the fastest time for loading the deep learning model, at 3.7 seconds. This is because the server has the largest GPU memory compared to the others, so the process of model reading is faster.

The last aspect tested is the throughput time of the server. In this test, the reliability of the four server specifications in serving inference requests is assessed. The length of time used in testing the throughput time is one second. Figure 6 shows that servers with CPU $2+$ GPU 2 have the largest number of services, at 335 . This is directly proportional to the total inference time, which only takes 0.0079 seconds to predict input data (Figure 4). For single ECG signal prediction, the dominating delay is the 1D-CNNs model loading and neural network setup, with the actual inference being comparatively fast on all frameworks and with both models. With the advance of GPU technology, our DL model can approximate a very complicated learning function with a reasonable training time. 1D-CNNs can make predictions directly from raw data; hence the effectiveness of the learning process is increased when large datasets are available. 


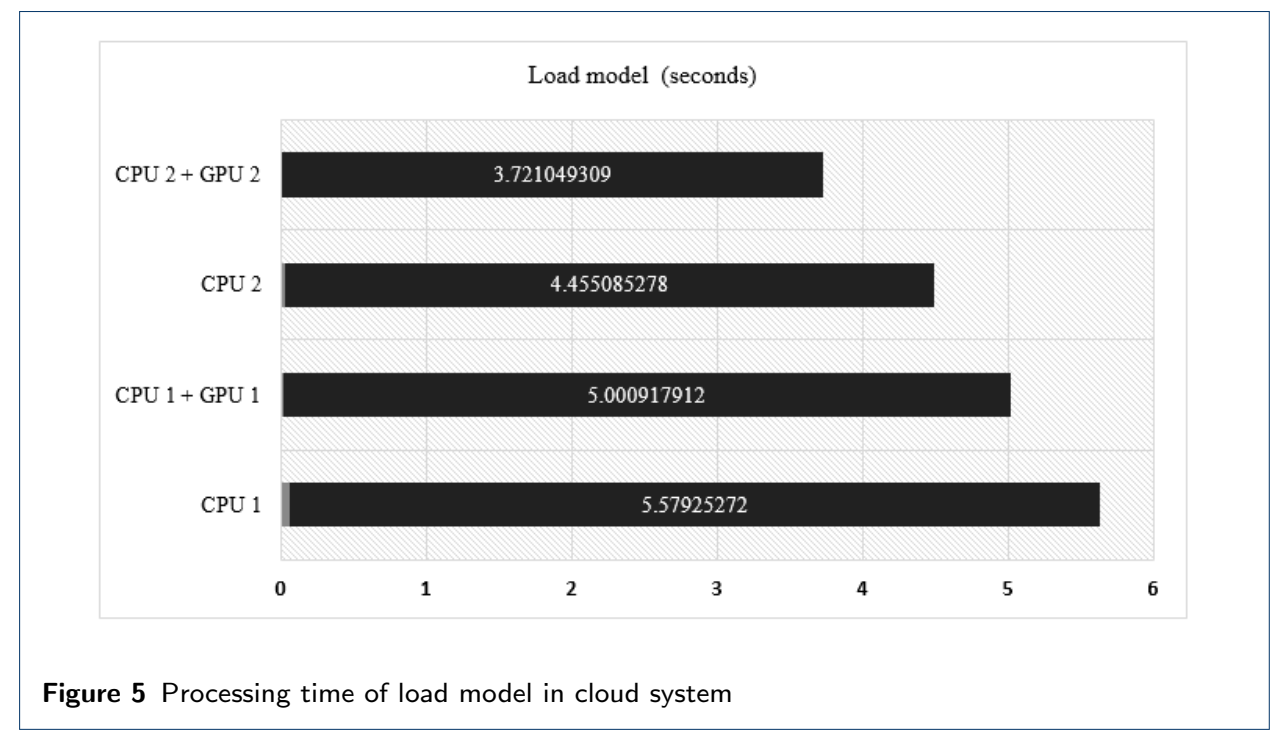

Figure 5 Processing time of load model in cloud system

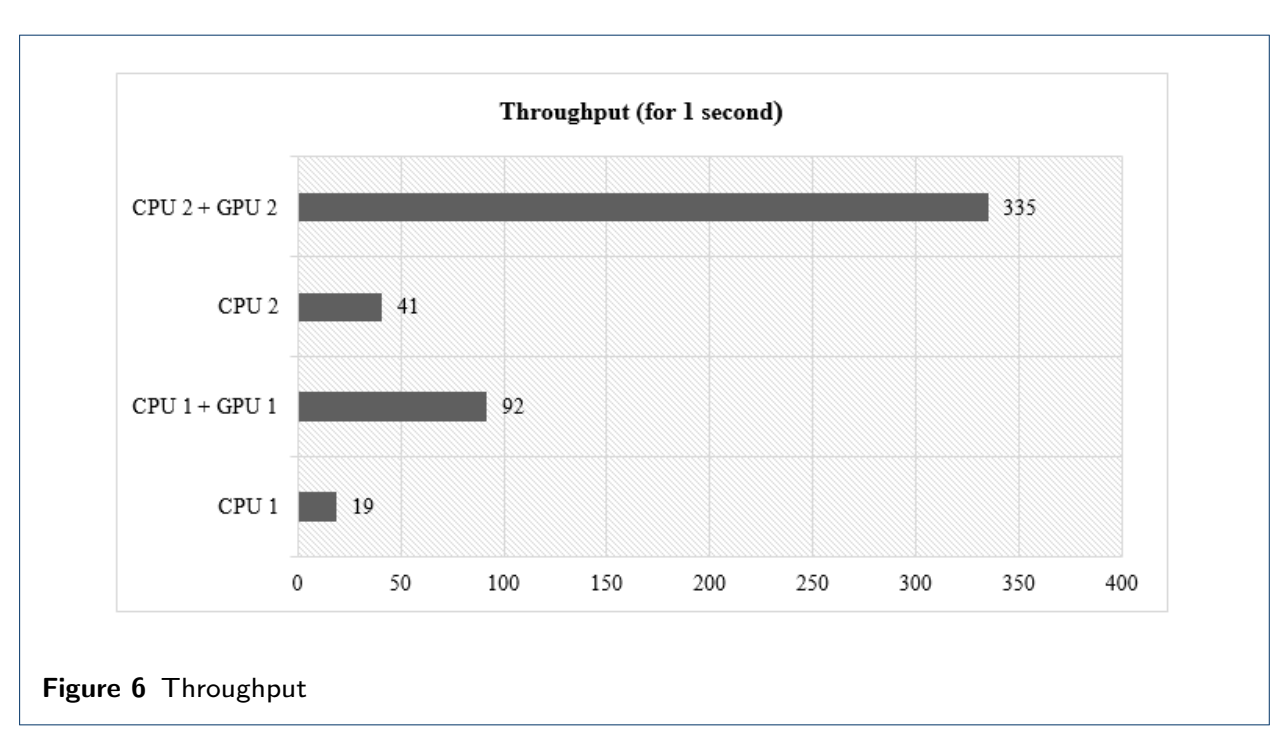

\section{Benchmarking Over Other DL Algorithms with the Cloud System}

This study achieved $100 \%$ accuracy for unseen data testing of $\mathrm{N}$ and AF with different frequency samplings and datasets (refer to Table 5). The proposed 1DCNNs model obtained robust performance under several conditions. For clinical use, the AI-aided ECG AF diagnostic method we developed appears to be sufficiently accurate. For major general hospitals, it may help to minimize misdiagnosis, thereby saving labor costs. This study has also solved the ECG signal problem regarding unequal signal lengths, frequency sampling, and imbalanced data. This study has also compared the previous studies that used both limited and large ECG datasets.

Some previous studies have explored the performance of the cloud for AF detection based on deep learning approaches, such as autoencoders, CNNs, and LSTMs (refer to Table 8). For example, Faust et al. [4] detected episodes of AF using heart rate signals and RNNs with a LSTM model. The RNNs with the LSTM model provided the intelligence needed for state-of-the-art IoT-based diagnosis support systems. 
They trained and tested with labeled HR signal data from 20 subjects sourced from PhysioNet Atrial Fibrillation Database (AFDB) and blindfolded validation, using the data from 3 subjects from AFDB and 82 subjects sourced from the Long-Term AF Database. Both performances of the dataset achieved $99.77 \%$ and $94 \%$ accuracy. Torres-Soto et al. [51] evaluated the use of convolutional denoising autoencoders for unsupervised learning as a pretraining technique, part of a hybrid approach where pretrained weights were used in the foundational layers of DeepBeat. They implemented the ambulatory monitoring dataset and correctly detected AF presence with $98 \%$ sensitivity and $99 \%$ specificity. Hong et al. [52] introduced their work on building, training, and serving an out-of-the-box cloud deep learning service they called CardioLearn for cardiac disease detection from ECGs. They used the ECG data with two forms of input: single lead and 12-lead. They tested CNNRNN as the proposed approach on the 2018 China Physiological Signal Challenge dataset and achieved $98.57 \%$ and $97.89 \%$ receiver operating characteristics (ROC) and the area under the ROC (ROC-AUC) scores for single lead and 12-lead data. They also designed a portable smart hardware device, along with an interactive mobile program, to demonstrate its practical use. Zhang et al. [53] established the Cardiovascular Disease Whole Process Management Platform for automated detection and classification of ECG signals. They obtained $98.27 \%$ accuracy for recognition of 18 classes of heart rhythms based on a CNNs model. Their proposed model also achieved $99.95 \%$ sensitivity for AF detection and $85.49 \%$ accuracy and $88.52 \%$ sensitivity for normal cases. Yildirim et al. [54] proposed an effective deep neural networks (DNNs) to detect different rhythm classes from ECG databases. With the 1,780 AF and 1,825 normal cases, the class-based performance achieved an average $97.91 \%$ accuracy, $96.52 \%$ sensitivity, and $98.31 \%$ specificity from Lead II-inputs for AF and normal sinus detection.

In the present study, we have proposed a one-dimensional CNNs for AF detection. We have experimented the testing (unseen data) with and without ECG recordings from Chapman University and Shaoxing People's Hospital. Without the database, total records of 8,416 were achieved with $100 \%$ accuracy, sensitivity, and specificity. We then added more data for 11,842 subjects and obtained $98.94 \%$ accuracy, $98.97 \%$ sensitivity, and $98.97 \%$ specificity. Although the performance results were decreased, the proposed model was still reliable for AF detection.

Table 8 Benchmarking with other DL for AF detection

\begin{tabular}{|c|c|c|c|c|c|c|}
\hline Author & Method & $\begin{array}{l}\text { Total } \\
\text { Subject }\end{array}$ & $\begin{array}{l}\text { Acc. } \\
(\%)\end{array}$ & $\begin{array}{c}\text { Sens. } \\
(\%)\end{array}$ & $\begin{array}{c}\text { Spec. } \\
(\%)\end{array}$ & $\begin{array}{l}\text { ROC AUC } \\
\text { score }(\%)\end{array}$ \\
\hline Faust et al. [4] & RNNs-LSTM & 102 & 98.51 & - & - & - \\
\hline $\begin{array}{l}\text { Torres-soto et al. } \\
\text { [51] }\end{array}$ & $\begin{array}{l}\text { Convolutional } \\
\text { Denoising } \\
\text { Autoencoders }\end{array}$ & 162 & - & 98 & 99 & - \\
\hline Hong et al. [52] & CNNs-RNNs & $\pm 20,000$ & - & - & - & 98.57 \\
\hline Zhang et al. [53] & CNNs & 177,941 & 91.88 & 94.23 & - & - \\
\hline Yildirim et al. [54] & DNNs & 3,605 & 97.91 & 96.52 & 98.31 & - \\
\hline Proposed model & 1D-CNNs & 8,416 & 100 & 100 & 100 & - \\
\hline & & 11,842 & 98.94 & 98.97 & 98.97 & - \\
\hline
\end{tabular}

In summary, we demonstrate that an end-to-end approach using 1D-CNNs will classify AF from single-lead ECGs from a wide variety of separate instruments with a diagnostic efficiency close to that of cardiologists. This method, if verified 
in clinical settings, has the potential to enhance the precision, performance, and scalability of ECG interpretation. However, our generalization model of a 1D-CNNs also has some limitations, which can be summarized as follows:

1 Our method is tested only for $\mathrm{N}$ and $\mathrm{AF}$ detection, whereas a wide variety of different arrhythmias detected from single-lead ECGs need to be classified in the future and researched in depth to confirm a high diagnostic output close to that of cardiologists.

2 The proposed single learning method will be tailored to the target application prior to clinical application, which could entail additional pre- or postprocessing steps.

3 Our DL-cloud architecture, which was not focused on the calculations of actual workloads and real computing platforms, was only available for neural network inference in terms of software frameworks and hardware acceleration.

\section{Conclusion}

AF has a high risk of severe health consequences, including death and stroke. Therefore, continuous AF monitoring could have a beneficial clinical impact by allowing the identification of AF in patients with post-ablation chronic AF or pharmacological cardioversion, for example. Our study is the first comprehensive demonstration of a DL approach to perform classification across a broad range of the most common and important ECG rhythm diagnoses with large datasets. We highlight the differences in the length of the ECG recording, the frequency sampling, and the data acquisition devices. This highlights the ability of our end-to-end 1D-CNNs-based approach to generalize the cloud deep-learning approach to a new set of AF rhythm labels on a number of datasets.

The approach exhibited rapid adoption that provides a chance for highly scalable AF detection. In the current study, we have trained and validated varied data with different frequency sampling. We also used unseen data from public and Indonesian hospital datasets to measure the robustness of proposed model. All ECG recordings were segmented into 2700 samples, which can present up to two R-R intervals. The 1D-CNNs model with 13 convolutions and 5 max-pooling layers reached the classification performance of $99.80 \%$ accuracy, sensitivity, and specificity in the training and validation data. The unseen data from 3,610 records used as blindfold validation revealed that the model achieved $98.94 \%$ accuracy, $98.97 \%$ sensitivity, and $98.97 \%$ specificity.

We also tested the scalability of the proposed model for different server specifications, such as a cloud server. The AFibNet was capable of generating an AF prediction quickly, indicating that our DL-based 1D-CNNs model has outstanding performance results. For functional diagnostic assistance, this concept is important since using information gained over a limited training period is precisely what a cardiologist does. In the future, the recommended model could be used to better classify AF patients early on, so that they can be managed to avoid stroke.

\section{Declarations}


Consent for publication

Not applicable

Availability of data and materials

All the data considered for this study is available at PhysioNet, https://physionet.org/

Competing interests

The authors declare that they have no competing interests

Funding

This work was supported by Basic Research Grants (096/SP2H/LT/DRPM/2019) from the Ministry of Research, Technology, and Higher Education, Indonesia and Competitive Grant 2020 from Universitas Sriwijaya Indonesia.

Authors' contributions

B.T. Conceptualization, Conceived and designed the analysis, Data Curation, Investigation. S.N. Conceptualization, Wrote the manuscript, Performed the analysis, Funding acquisition, Formal Analysis, Methodology. A.E.T. Medical Validation. M.N.R. Designing computer programs, Formal Analysis, Methodology. A.D. Wrote the manuscript, Contributed data or analysis tools, Formal Analysis, Methodology, Data curation. R.E. Designing computer programs, Data curation. F.F. Resources, Data Curation. A.I.S. Visualization Preparation.

Acknowledgements

We thank to the Intelligent Systems Research Group (ISysRG), Universitas Sriwijaya.

Author details

${ }^{1}$ Intelligent System Research Group, Faculty of Computer Science, Universitas Sriwijaya, Palembang, 30139,

Indonesia. ${ }^{2}$ Department of Cardiology Vascular Medicine, Dr. Mohammad Hoesin Hospital, Palembang, Indonesia.

References

1. De Chazal, P., O'Dwyer, M., Reilly, R.B.: Automatic classification of heartbeats using ecg morphology and heartbeat interval features. IEEE transactions on biomedical engineering 51(7), 1196-1206 (2004)

2. Mant, J., Fitzmaurice, D.A., Hobbs, F.R., Jowett, S., Murray, E.T., Holder, R., Davies, M., Lip, G.Y.: Accuracy of diagnosing atrial fibrillation on electrocardiogram by primary care practitioners and interpretative diagnostic software: analysis of data from screening for atrial fibrillation in the elderly (safe) trial. Bmj 335(7616), 380 (2007)

3. Tondas, A.E., Halim, R.A., Guyanto, M.: Minimal or no touch electrocardiography recording and remote heart rhythm monitoring during covid-19 pandemic era. Indonesian Journal of Cardiology 41(2), 133-41 (2020)

4. Faust, O., Kareem, M., Shenfield, A., Ali, A., Acharya, U.R.: Validating the robustness of an internet of things based atrial fibrillation detection system. Pattern Recognition Letters 133, 55-61 (2020)

5. Pranata, R., Yonas, E., Chintya, V., Tondas, A.E., Raharjo, S.B.: Evidence-based case report: The use of d-dimer assay to exclude left atrial thrombus in patient with atrial fibrillation $i 48$ hours. Journal of atrial fibrillation 11(6) (2019)

6. Pranata, R., Tondas, A.E., Yonas, E., Chintya, V., Yamin, M.: Efficacy and safety of catheter ablation for atrial fibrillation in congenital heart disease-a systematic review and meta-analysis. Indian pacing and electrophysiology journal 19(6), 216-221 (2019)

7. Yuniadi, Y., Hanafy, D.A., Rahardjo, S.B., Tondas, A.E., Maharani, E., Hermanto, D.Y., Munawar, M.: 2014 indonesian heart association guidelines of management of atrial fibrillation. Indonesian Journal of Cardiology, 102-33 (2014)

8. Shah, A.P., Rubin, S.A.: Errors in the computerized electrocardiogram interpretation of cardiac rhythm. Journal of electrocardiology 40(5), 385-390 (2007)

9. Bowry, A.D., Lewey, J., Dugani, S.B., Choudhry, N.K.: The burden of cardiovascular disease in low-and middle-income countries: epidemiology and management. Canadian Journal of Cardiology 31(9), 1151-1159 (2015)

10. Ribeiro, A.H., Ribeiro, M.H., Paixão, G.M., Oliveira, D.M., Gomes, P.R., Canazart, J.A., Ferreira, M.P., Andersson, C.R., Macfarlane, P.W., Meira Jr, W., et al.: Automatic diagnosis of the 12-lead ecg using a deep neural network. Nature communications 11(1), 1-9 (2020)

11. Nurmaini, S., Umi Partan, R., Caesarendra, W., Dewi, T., Naufal Rahmatullah, M., Darmawahyuni, A., Bhayyu, V., Firdaus, F.: An automated ecg beat classification system using deep neural networks with an unsupervised feature extraction technique. Applied Sciences 9(14), 2921 (2019)

12. Tison, G.H., Sanchez, J.M., Ballinger, B., Singh, A., Olgin, J.E., Pletcher, M.J., Vittinghoff, E., Lee, E.S., Fan, S.M., Gladstone, R.A., et al.: Passive detection of atrial fibrillation using a commercially available smartwatch. JAMA cardiology 3(5), 409-416 (2018)

13. Nurmaini, S., Tondas, A.E., Darmawahyuni, A., Rachmatullah, M.N., Partan, R.U., Firdaus, F., Tutuko, B., Pratiwi, F., Juliano, A.H., Khoirani, R.: Robust detection of atrial fibrillation from short-term electrocardiogram using convolutional neural networks. Future Generation Computer Systems 113, 304-317 (2020)

14. Faust, O., Ciaccio, E.J., Acharya, U.R.: A review of atrial fibrillation detection methods as a service. International journal of environmental research and public health 17(9), 3093 (2020)

15. Ebrahimi, Z., Loni, M., Daneshtalab, M., Gharehbaghi, A.: A review on deep learning methods for ecg arrhythmia classification. Expert Systems with Applications: X, 100033 (2020)

16. Andersen, R.S., Peimankar, A., Puthusserypady, S.: A deep learning approach for real-time detection of atrial fibrillation. Expert Systems with Applications 115, 465-473 (2019)

17. Faust, O., Hagiwara, Y., Hong, T.J., Lih, O.S., Acharya, U.R.: Deep learning for healthcare applications based on physiological signals: A review. Computer methods and programs in biomedicine 161, 1-13 (2018) 
18. Darmawahyuni, A., Nurmaini, S., Yuwandini, M., Rachmatullah, M.N., Firdaus, F., Tutuko, B.: Congestive heart failure waveform classification based on short time-step analysis with recurrent network. Informatics in Medicine Unlocked 21, 100441 (2020)

19. Farhadi, J., Attarodi, G., Dabanloo, N.J., Mohandespoor, M., Eslamizadeh, M.: Classification of atrial fibrillation using stacked auto encoders neural networks. In: 2018 Computing in Cardiology Conference (CinC), vol. 45, pp. 1-3 (2018). IEEE

20. Erdenebayar, U., Kim, H., Park, J.-U., Kang, D., Lee, K.-J.: Automatic prediction of atrial fibrillation based on convolutional neural network using a short-term normal electrocardiogram signal. Journal of Korean medical science 34(7) (2019)

21. Cai, W., Chen, Y., Guo, J., Han, B., Shi, Y., Ji, L., Wang, J., Zhang, G., Luo, J.: Accurate detection of atrial fibrillation from 12-lead ecg using deep neural network. Computers in biology and medicine 116, 103378 (2020)

22. Liaqat, S., Dashtipour, K., Zahid, A., Assaleh, K., Arshad, K., Ramzan, N.: Detection of atrial fibrillation using a machine learning approach. Information 11(12), 549 (2020)

23. Zhou, X., Zhu, X., Nakamura, K., Noro, M.: Atrial fibrillation detection using convolutional neural networks. In: 2018 9th International Conference on Awareness Science and Technology (iCAST), pp. 84-89 (2018). IEEE

24. Huang, M.-L., Wu, Y.-S.: Classification of atrial fibrillation and normal sinus rhythm based on convolutional neural network. Biomedical Engineering Letters, 1-11 (2020)

25. Al Rahhal, M.M., Bazi, Y., Al Zuair, M., Othman, E., BenJdira, B.: Convolutional neural networks for electrocardiogram classification. Journal of Medical and Biological Engineering 38(6), 1014-1025 (2018)

26. Kiranyaz, S., Ince, T., Gabbouj, M.: Real-time patient-specific ecg classification by 1-d convolutional neural networks. IEEE Transactions on Biomedical Engineering 63(3), 664-675 (2015)

27. Acharya, U.R., Oh, S.L., Hagiwara, Y., Tan, J.H., Adam, M., Gertych, A., San Tan, R.: A deep convolutional neural network model to classify heartbeats. Computers in biology and medicine 89, 389-396 (2017)

28. Hannun, A.Y., Rajpurkar, P., Haghpanahi, M., Tison, G.H., Bourn, C., Turakhia, M.P., Ng, A.Y.: Cardiologist-level arrhythmia detection and classification in ambulatory electrocardiograms using a deep neural network. Nature medicine 25(1), 65-69 (2019)

29. Li, Y., Pang, Y., Wang, J., Li, X.: Patient-specific ecg classification by deeper cnn from generic to dedicated Neurocomputing 314, 336-346 (2018)

30. Oh, S.L., Ng, E.Y., San Tan, R., Acharya, U.R.: Automated beat-wise arrhythmia diagnosis using modified u-net on extended electrocardiographic recordings with heterogeneous arrhythmia types. Computers in biology and medicine 105, 92-101 (2019)

31. Subasi, A., Qaisar, S.M.: Heartbeat classification using parametric and time-frequency methods

32. Li, F., Wu, J., Jia, M., Chen, Z., Pu, Y.: Automated heartbeat classification exploiting convolutional neural network with channel-wise attention. IEEE Access 7, 122955-122963 (2019)

33. Yıldırım, Ö., Pławiak, P., Tan, R.-S., Acharya, U.R.: Arrhythmia detection using deep convolutional neural network with long duration ecg signals. Computers in biology and medicine 102, 411-420 (2018)

34. Shaker, A.M., Tantawi, M., Shedeed, H.A., Tolba, M.F.: Generalization of convolutional neural networks for ecg classification using generative adversarial networks. IEEE Access 8, 35592-35605 (2020)

35. Hamon, R., Junklewitz, H., Sanchez, I.: Robustness and explainability of artificial intelligence. Publications Office of the European Union (2020)

36. Jagadeeswari, V., Subramaniyaswamy, V., Logesh, R., Vijayakumar, V.: A study on medical internet of things and big data in personalized healthcare system. Health information science and systems 6(1), 1-20 (2018)

37. Alkmim, M.B., Figueira, R.M., Marcolino, M.S., Cardoso, C.S., Abreu, M.P.d., Cunha, L.R., Cunha, D.F.d., Antunes, A.P., Resende, A.G.d.A., Resende, E.S., et al.: Improving patient access to specialized health care: the telehealth network of minas gerais, brazil. Bulletin of the World Health Organization 90, 373-378 (2012)

38. Draghici, A.E., Taylor, J.A.: The physiological basis and measurement of heart rate variability in humans. Journal of Physiological Anthropology 35(1), 1-8 (2016)

39. Moody, G.: A new method for detecting atrial fibrillation using rr intervals. Computers in Cardiology, 227-230 (1983)

40. Goldberger, A.L., Amaral, L.A., Glass, L., Hausdorff, J.M., Ivanov, P.C., Mark, R.G., Mietus, J.E., Moody, G.B., Peng, C.-K., Stanley, H.E.: Physiobank, physiotoolkit, and physionet: components of a new research resource for complex physiologic signals. circulation 101(23), 215-220 (2000)

41. Liu, F., Liu, C., Zhao, L., Zhang, X., Wu, X., Xu, X., Liu, Y., Ma, C., Wei, S., He, Z., et al.: An open access database for evaluating the algorithms of electrocardiogram rhythm and morphology abnormality detection. Journal of Medical Imaging and Health Informatics 8(7), 1368-1373 (2018)

42. Petrutiu, S., Sahakian, A.V., Swiryn, S.: Abrupt changes in fibrillatory wave characteristics at the termination of paroxysmal atrial fibrillation in humans. Europace 9(7), 466-470 (2007)

43. Moody, G.: Spontaneous termination of atrial fibrillation: a challenge from physionet and computers in cardiology 2004. In: Computers in Cardiology, 2004, pp. 101-104 (2004). IEEE

44. lyengar, N., Peng, C., Morin, R., Goldberger, A.L., Lipsitz, L.A.: Age-related alterations in the fractal scaling of cardiac interbeat interval dynamics. American Journal of Physiology-Regulatory, Integrative and Comparative Physiology 271(4), 1078-1084 (1996)

45. Moody, G.B., Mark, R.G.: The impact of the mit-bih arrhythmia database. IEEE Engineering in Medicine and Biology Magazine 20(3), 45-50 (2001)

46. Zheng, J., Zhang, J., Danioko, S., Yao, H., Guo, H., Rakovski, C.: A 12-lead electrocardiogram database for arrhythmia research covering more than 10,000 patients. Scientific data $7(1), 1-8(2020)$

47. Ahmed, N., Zhu, Y.: Early detection of atrial fibrillation based on ecg signals. Bioengineering 7(1), 16 (2020)

48. Mahdiani, S., Jeyhani, V., Peltokangas, M., Vehkaoja, A.: Is 50 hz high enough ecg sampling frequency for accurate hrv analysis? In: 2015 37th Annual International Conference of the IEEE Engineering in Medicine and Biology Society (EMBC), pp. 5948-5951 (2015). IEEE

49. Kwon, O., Jeong, J., Kim, H.B., Kwon, I.H., Park, S.Y., Kim, J.E., Choi, Y.: Electrocardiogram sampling frequency range acceptable for heart rate variability analysis. Healthcare informatics research 24(3), 198 (2018) 
50. Lim, K., Ranganathan, P., Chang, J., Patel, C., Mudge, T., Reinhardt, S.: Understanding and designing new server architectures for emerging warehouse-computing environments. ACM SIGARCH Computer Architecture News 36(3), 315-326 (2008)

51. Torres-Soto, J., Ashley, E.A.: Multi-task deep learning for cardiac rhythm detection in wearable devices. NPJ digital medicine 3(1), 1-8 (2020)

52. Hong, S., Fu, Z., Zhou, R., Yu, J., Li, Y., Wang, K., Cheng, G.: Cardiolearn: A cloud deep learning service for cardiac disease detection from electrocardiogram. In: Companion Proceedings of the Web Conference 2020, pp. $148-152(2020)$

53. Zhang, X., Gu, K., Miao, S., Zhang, X., Yin, Y., Wan, C., Yu, Y., Hu, J., Wang, Z., Shan, T., et al.: Automated detection of cardiovascular disease by electrocardiogram signal analysis: a deep learning system. Cardiovascular Diagnosis and Therapy 10(2), 227 (2020)

54. Yildirim, O., Talo, M., Ciaccio, E.J., San Tan, R., Acharya, U.R.: Accurate deep neural network model to detect cardiac arrhythmia on more than 10,000 individual subject ecg records. Computer methods and programs in biomedicine 197, 105740 (2020) 


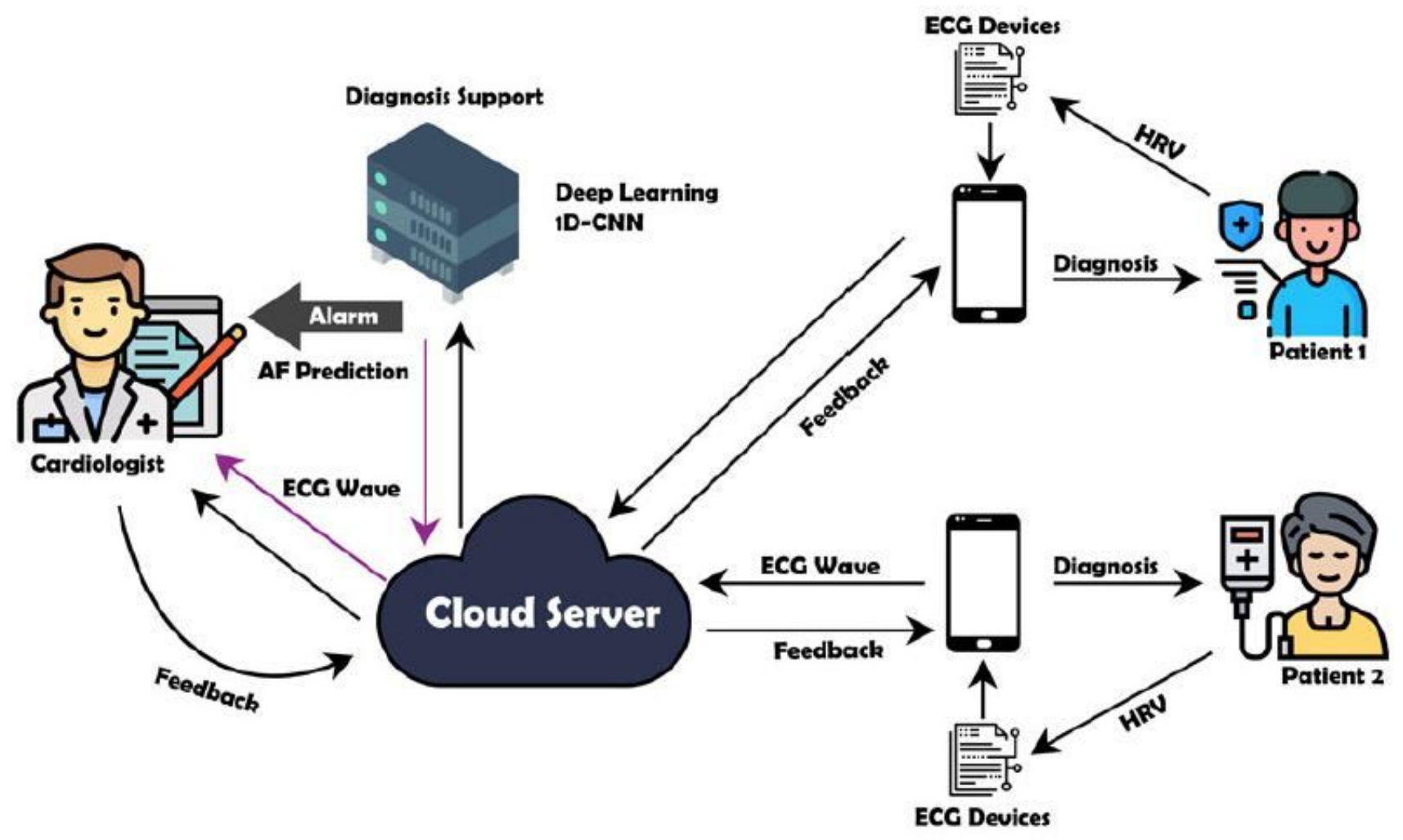

Figure 1

Flow diagram of deep learning model for AF diagnosis system 

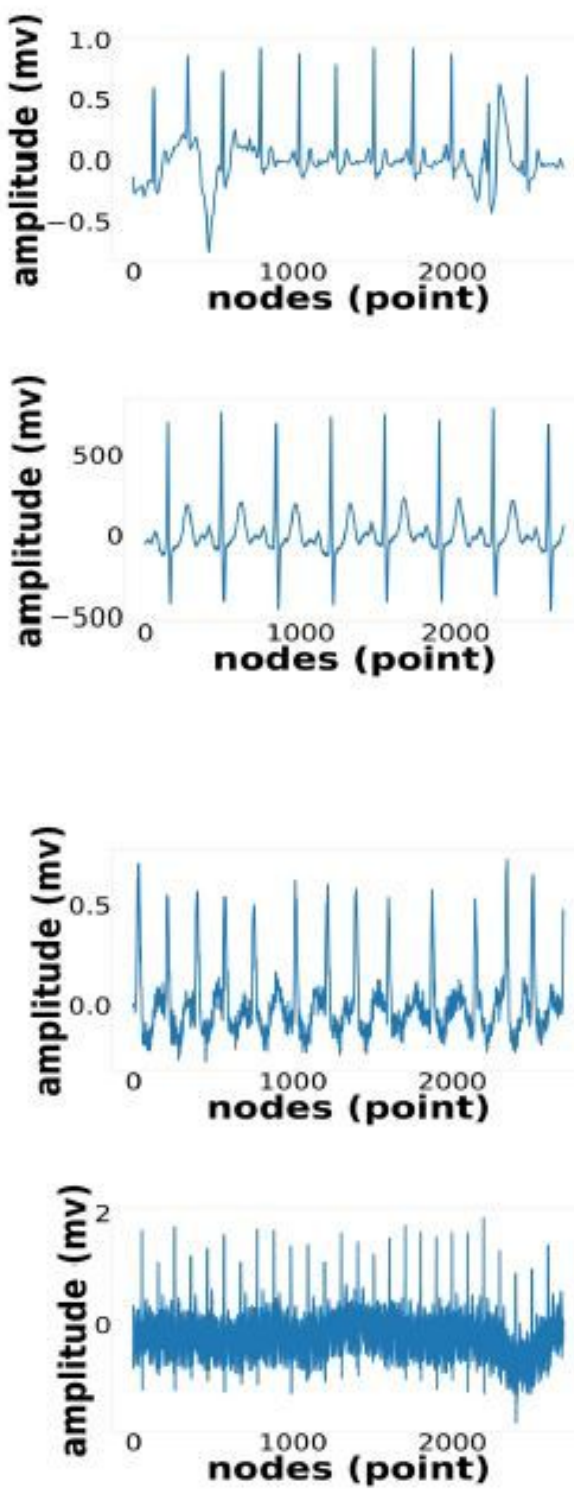
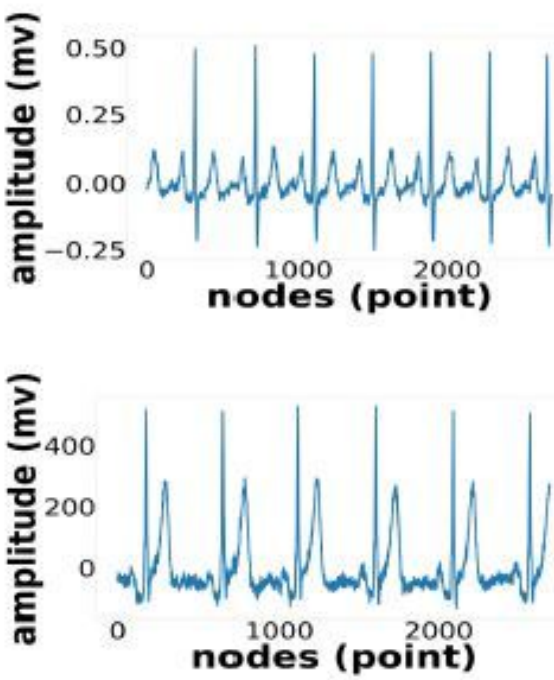

(a) Normal Sinus
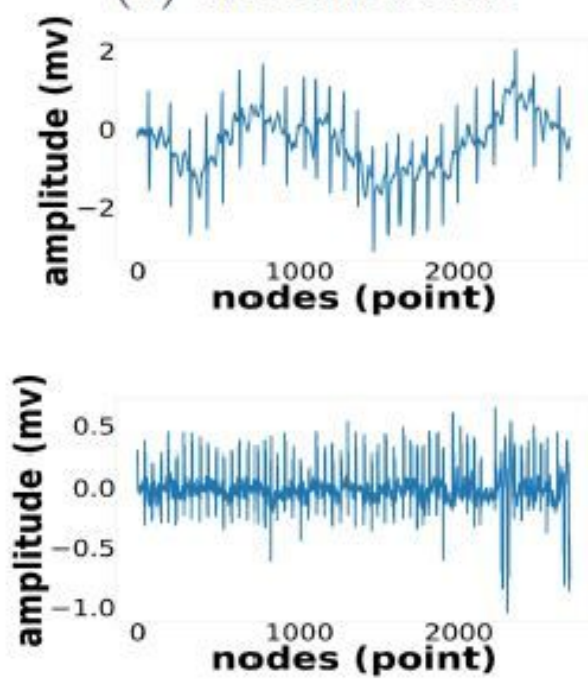

(b) Atrial Fibrillation
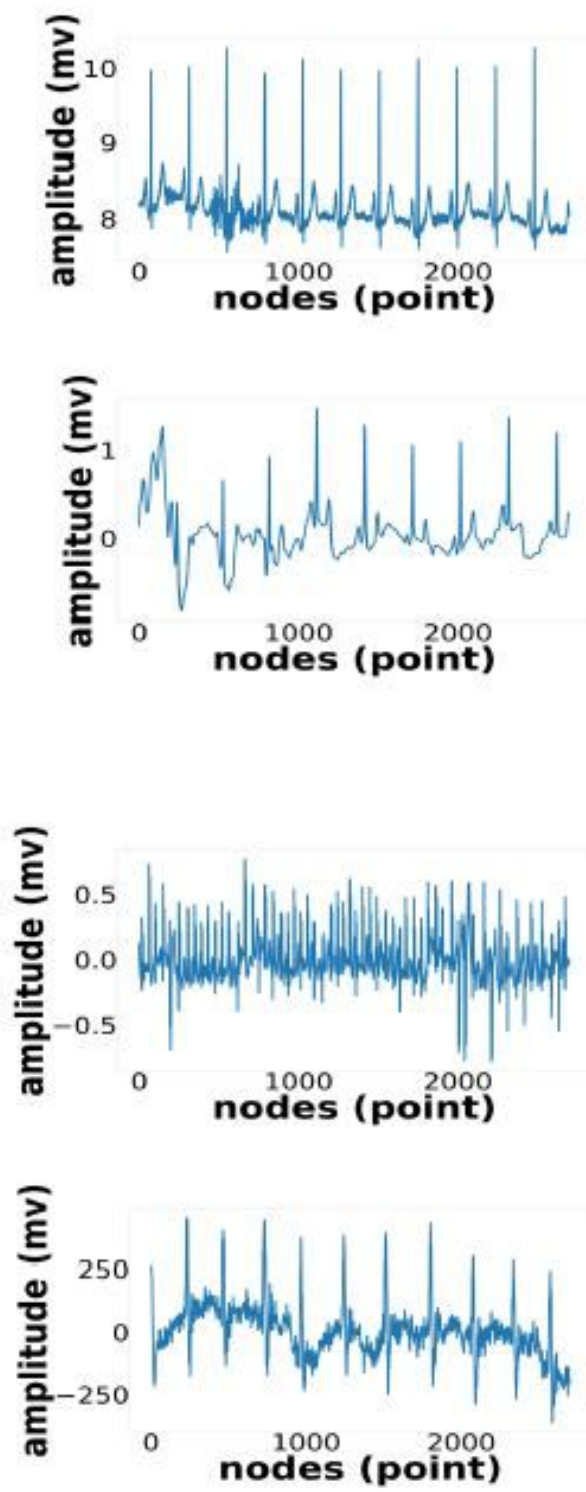

Figure 2

Flow diagram of deep learning model for AF diagnosis system 
Noise Removal Sym5 (Discrete Wavelet Transform)

Record Standard Single-lead ECG Raw Data

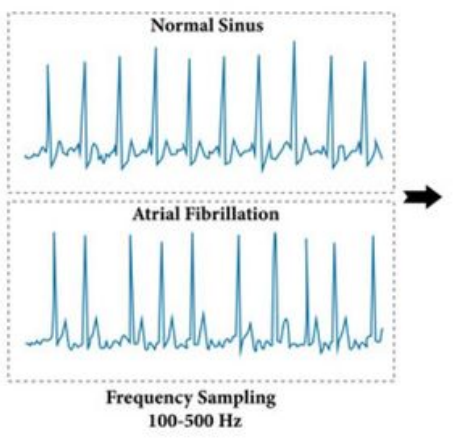

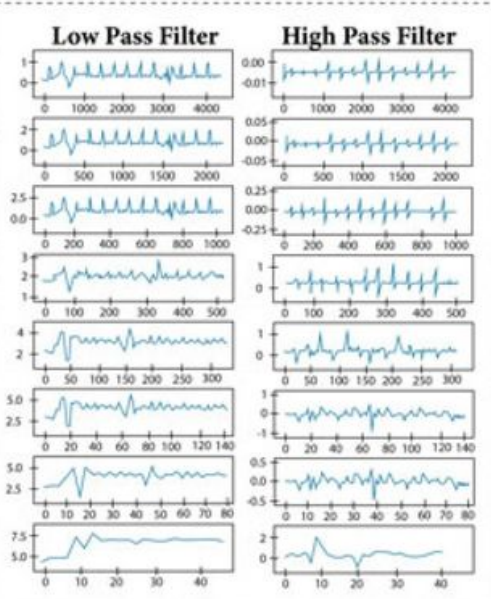

Segmentation of Rhythm Episodes for 2700 Nodes

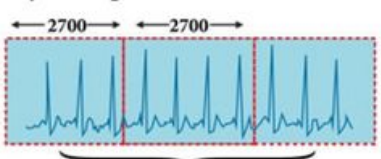

ECG signal segments to each 2700 samples for all records
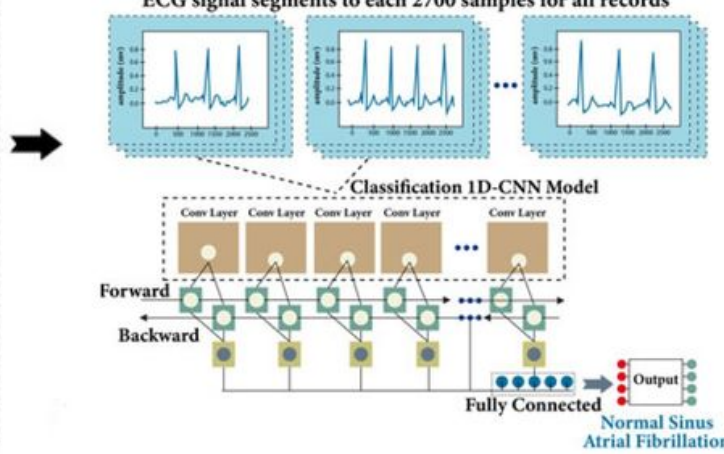

Figure 3

Proposed the AFibNet for explainable Al methodology

\section{Processing time (seconds)}

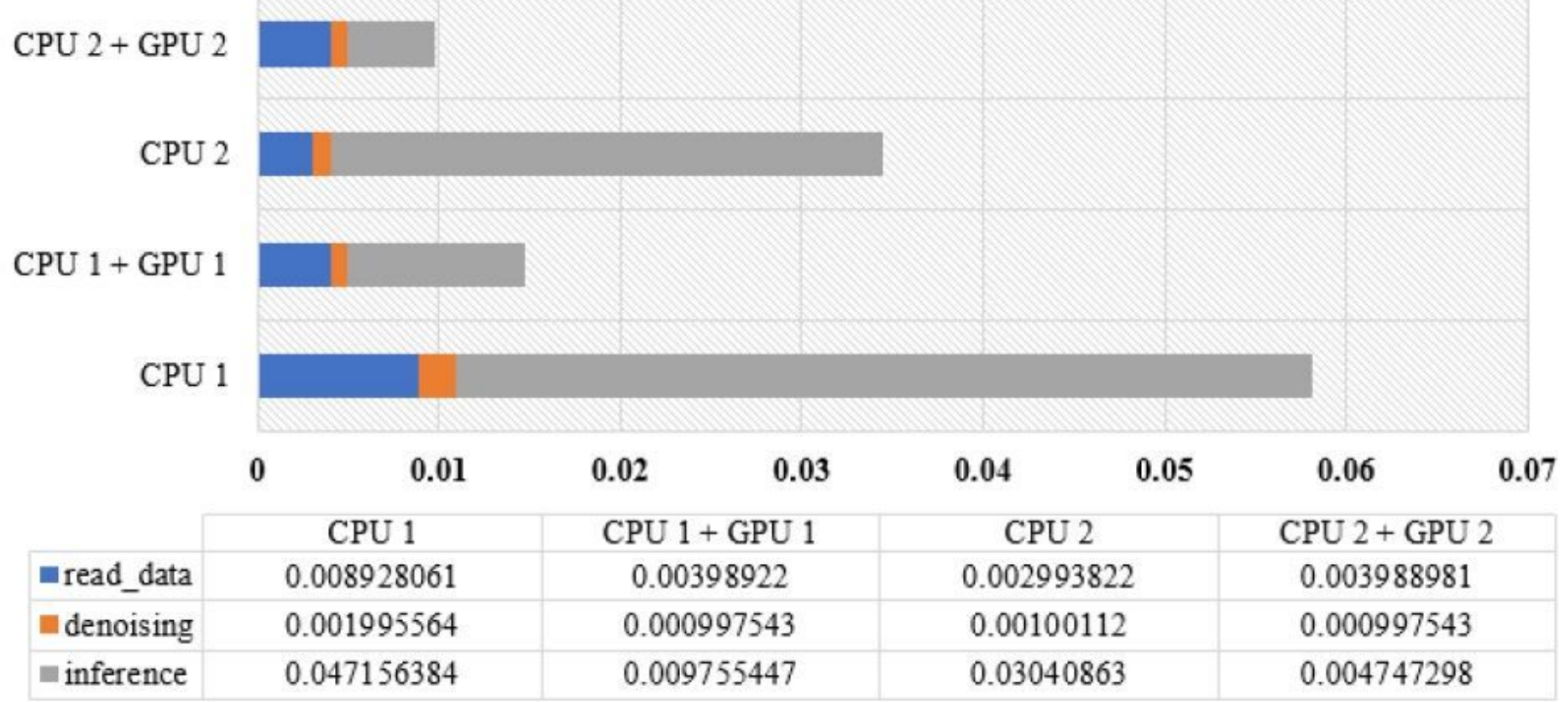

Figure 4

Processing time of 1D-CNNs in four server specication 


\section{Load model (seconds)}

CPU 2 + GPU 2

\subsection{9}

CPU 2

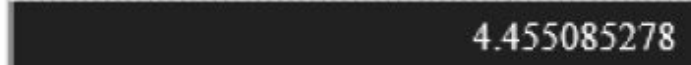

CPU 1 + GPU 1

\subsection{2}

CPU 1

5.57925272

0

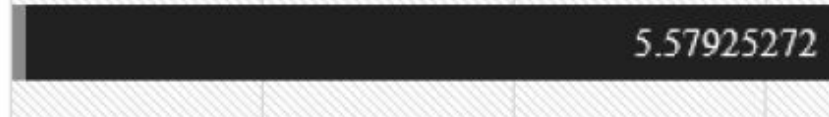

1

2

3

4

5

Figure 5

Processing time of load model in cloud system

Throughput (for 1 second)

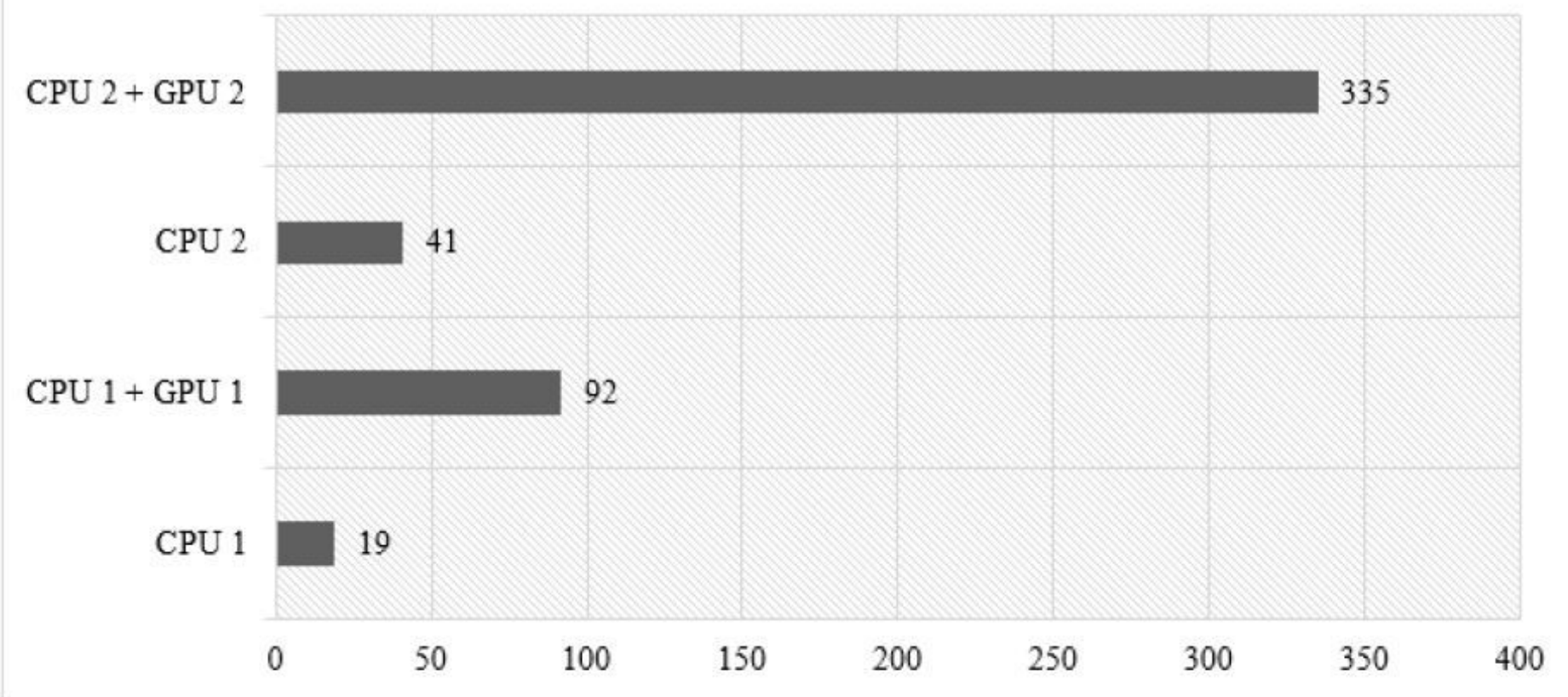

Figure 6 
Throughput 